Marek Fila $\cdot$ Michael Winkler

\title{
Single-point blow-up on the boundary where the zero Dirichlet boundary condition is imposed
}

Received June 21, 2006 and in revised form September 20, 2006

Abstract. We consider a reaction-diffusion-convection equation on the halfline $(0, \infty)$ with the zero Dirichlet boundary condition at $x=0$. We find a positive selfsimilar solution $u$ which blows up in a finite time $T$ at $x=0$ while $u(x, T)$ remains bounded for $x>0$.

\section{Introduction}

We study the existence of positive backward selfsimilar solutions of the problem

$$
\left\{\begin{array}{l}
u_{t}=u_{x x}+k\left(u^{m}\right)_{x}+u^{2 m-1}, \quad x \in(0, \infty), t \in(-\infty, T), \\
u(0, t)=0, \quad t \in(-\infty, T),
\end{array}\right.
$$

where $k>0, m>1$ and $T \in \mathbb{R}$. More precisely, we look for solutions of the form

$$
u(x, t)=(T-t)^{-1 / 2(m-1)} w(y), \quad y=(T-t)^{-1 / 2} x,
$$

where $w$ is a solution of the problem

$$
\left\{\begin{array}{l}
w^{\prime \prime}=\frac{y}{2} w^{\prime}-k\left(w^{m}\right)^{\prime}+\frac{1}{2(m-1)} w-w^{2 m-1}, \quad y>0 \\
w(0)=0
\end{array}\right.
$$

It follows from [9] that if $k=0$ then there is no bounded nontrivial nonnegative solution of (1.3).

Our main aim is to show the following:

Theorem 1.1. Let $m>1$. Then there is $k_{0}>2 / \sqrt{m}$ such that for any $k \geq k_{0}$ the problem 1.3 possesses a positive solution satisfying $\lim _{\sup } \rightarrow \infty y^{1 /(m-1)} w(y)<\infty$. Consequently, for any $T \in \mathbb{R}$ the function u defined by $(1.2)$ is a positive classical solution of (1.1) such that $\lim _{t \nearrow T}\|u(\cdot, t)\|_{L^{\infty}((0, \infty))}=\infty$. Moreover, there is a constant $C>0$ such that $u(x, t) \leq C x^{-1 /(m-1)}$ for $x>0$ and $t<T$.

M. Fila: Department of Applied Mathematics and Statistics, Comenius University, Mlynská dolina, 84248 Bratislava, Slovakia; e-mail: fila@fmph.uniba.sk

M. Winkler: Department of Mathematics I, RWTH Aachen, 52056 Aachen, Germany; e-mail: micha_win@gmx.de 
This means that the solution $u$ blows up at the single point $x=0$ where the homogeneous Dirichlet boundary condition is prescribed.

For the following problem without convection:

$$
\left\{\begin{array}{l}
u_{t}=\Delta u+f(u), \quad x \in \Omega, t>0 \\
u(0, t)=0, \quad t>0, \\
u(x, 0)=u_{0}(x) \geq 0, \quad x \in \Omega
\end{array}\right.
$$

where $f(u)=u^{p}, p>1$ and $\Omega \subset \mathbb{R}^{N}$ is bounded, the first examples of single point blow-up were given in [18] $(N=1, \Omega=(-L, L))$ and [8, 13] $(N \geq 1, \Omega=\{|x|<L\})$. In these examples, the solutions blow up at $x=0$. It was also shown in [8] that if $\Omega$ is convex then blow-up of solutions of (1.4) can only occur away from the boundary $\partial \Omega$.

There are examples of solutions of parabolic equations for which singularities occur on the boundary where the homogeneous Dirichlet boundary condition is imposed (see [5, 15], for instance) but in these examples some spatial derivatives become unbounded while the solution itself stays bounded. This phenomenon is called gradient blow-up and it occurs for equations of the form

$$
u_{t}=\Delta u+f(u, \nabla u)
$$

where $f(u, \nabla u)$ grows superquadratically in $\nabla u$.

It is also known that $\partial \Omega$ may be contained in the blow-up set $\mathcal{B}(u)$ defined as

$$
\begin{aligned}
\mathcal{B}(u)=\left\{x_{0} \in \bar{\Omega} \mid \text { there is a sequence }\left\{\left(x_{n}, t_{n}\right)\right\} \subset \Omega \times(0, T)\right. \text { with } \\
\left.\left(x_{n}, t_{n}\right) \rightarrow\left(x_{0}, T\right) \text { such that } u\left(x_{n}, t_{n}\right) \rightarrow \infty\right\},
\end{aligned}
$$

if $u$ is a solution of 1.4 with $f(u)=(u+2) \log ^{p}(u+2), 1<p \leq 2$ (see [11]). But here $\mathcal{B}(u)=\bar{\Omega}$ and this phenomenon is called global blow-up.

Examples of single-point blow-up (in $L^{\infty}$ ) on the boundary are only known in the case when blow-up is forced by boundary conditions: see [4] for the case of nonlinear Neumann boundary conditions and [14] for the case when nonhomogeneous (blowing up) Dirichlet boundary data are prescribed.

Blow-up of solutions of reaction-diffusion-convection equations of the form

$$
u_{t}=\Delta u+k \nabla\left(u^{m}\right)+u^{p}, \quad m, p>1,
$$

(or more general) was studied before in [1, 3, 6, 7, 12, 17], for instance, and it is known to occur if $p>m$. Backward selfsimilar subsolutions of (1.5) were found in [17].

The existence of radial backward selfsimilar positive solutions of the equation

$$
u_{t}=\Delta u-k|\nabla u|^{2 p /(p+1)}+u^{p}, \quad k>0, p>1,
$$

was established in [16] for $k$ and $p-1$ small enough.

Our method relies on some shooting arguments but it is not related to the methods used in [16] or [2]. We study the initial value problem

$$
\left\{\begin{array}{l}
w^{\prime \prime}=\frac{y}{2} w^{\prime}-k\left(w^{m}\right)^{\prime}+\frac{1}{2(m-1)} w-w^{2 m-1}, \quad y>0, w>0, \\
w(0)=0, \quad w^{\prime}(0)=\sigma>0 .
\end{array}\right.
$$


Our strategy is to show that for $\sigma$ small enough the solution does not have any local minimum (Section 4) while the set $\Sigma$ of $\sigma$ such that the corresponding solution possesses a local minimum is nonempty (Section 5). The solution corresponding to $\bar{\sigma}:=\inf \Sigma$ is then the solution we are looking for (Section 6). Section 2 is devoted to some elementary properties of solutions of 1.6 and Section 3 contains some comparison lemmas which are used later.

\section{Preliminaries}

We first collect some basic properties of solutions of 1.6 . For $\sigma>0$, let $w_{\sigma}:\left(0, y_{\max }(\sigma)\right)$ $\rightarrow(0, \infty)$ denote the maximally extended positive solution of $(1.6)$. Then it is easily verified that any $w_{\sigma}$ is a priori bounded above in bounded subintervals of $[0, \infty)$, so that $w_{\sigma}$ may cease to exist only by approaching the value zero at some finite value of $y$. In order to keep the notation and arguments as simple as possible, let us henceforth instead of (1.6) consider the extended problem

$$
\begin{aligned}
& w^{\prime \prime}=\frac{y}{2} w^{\prime}-k\left(w_{+}^{m}\right)^{\prime}+\frac{1}{2(m-1)} w-w_{+}^{2 m-1}, \quad y>0, \\
& w(0)=0, \quad w^{\prime}(0)=\sigma,
\end{aligned}
$$

where $w_{+}:=\max \{w, 0\}$ denotes the positive part of $w$. In fact, 2.1, 2.2 possesses a solution on all of $(0, \infty)$ whenever $\sigma>0$, and on $\left(0, y_{\max }(\sigma)\right)$ this solution coincides with that of 1.3 , corresponding to the same initial data. Therefore throughout the following we may as well let $w_{\sigma}$ denote the globally defined solution of [2.1, 2.2) without danger of confusion.

Although for $m<2$ the term $\left(w_{+}^{m}\right)^{\prime}=m w_{+}^{m-1} w^{\prime}$ is not locally Lipschitz continuous with respect to the couple $\left(w, w^{\prime}\right) \in \mathbb{R}^{2}$, the solutions of 2.1 enjoy the usual properties of uniqueness and continuous dependence on the initial data; this can be seen by introducing the new variable $W:=w^{\prime}+k w_{+}^{m}-\frac{y}{2} w$, which transforms the ODE in 2.1 to the system

$$
\begin{aligned}
w^{\prime} & =W-k w_{+}^{m}+\frac{y}{2} w, \\
W^{\prime} & =\left(\frac{1}{2(m-1)}-\frac{1}{2}\right) w-w_{+}^{2 m-1} .
\end{aligned}
$$

Now the nonlinearity on the right-hand side is continuously differentiable with respect to $(w, W) \in \mathbb{R}^{2}$.

We next observe that 2.1 has three explicit solutions. One of these is the constant solution

$$
w \equiv \mu:=\left(\frac{1}{2(m-1)}\right)^{1 / 2(m-1)},
$$

which has the following evident property: 
Lemma 2.1. Let $w$ be any solution of 2.1. If $w$ attains a local minimum at some $y_{1}>0$ then $0 \leq w\left(y_{1}\right) \leq \mu$, whereas if $w$ has a positive local maximum at $y_{1}$ then $w\left(y_{1}\right) \geq \mu$.

Since we assume $k>2 / \sqrt{m}$ throughout, we can moreover find two explicit singular solutions $w_{s}^{ \pm}$of 2.1 given by

$$
w_{s}^{ \pm}(y)=A^{ \pm} y^{-1 /(m-1)}, \quad y>0,
$$

where $A^{ \pm}$are the two positive roots of the equation

$$
A^{2 m-2}-\frac{k m}{m-1} A^{m-1}+\frac{m}{(m-1)^{2}}=0,
$$

that is, we have

$$
\left(A^{ \pm}\right)^{m-1}=\frac{k m \pm \sqrt{k^{2} m^{2}-4 m}}{2(m-1)} .
$$

Since the larger root $A^{+}$will frequently appear in what follows, we will from now on drop its superscript and simply write $A=A^{+}$.

Another elementary property of 2.1 concerns the possible limits of stabilizing solutions.

Lemma 2.2. Let $w$ be a solution of 2.1 and assume that $w(y) \rightarrow w_{\infty}$ as $y \rightarrow \infty$ with some $w_{\infty} \in \mathbb{R}$. Then either $w_{\infty}=0$ or $w_{\infty}=\mu$.

Proof. The hypothesis implies that $W \equiv w^{\prime}-k w_{+}^{m}+\frac{y}{2} w$ satisfies

$$
W^{\prime}(y) \rightarrow\left(\frac{1}{2(m-1)}-\frac{1}{2}\right) w_{\infty}-\left(w_{\infty}\right)_{+}^{2 m-1}=: c_{0}
$$

by 2.3 . Hence for all $v>0$ one can find $y_{v}>0$ such that $\left|w(y)-w_{\infty}\right|<v$ as well as $\left|W^{\prime}(y)-c_{0}\right|<v$ for all $y>y_{v}$. In particular,

$$
\begin{aligned}
& w(y) \geq w_{\infty}-v, \quad w^{m}(y) \leq\left(w_{\infty}+v\right)_{+}^{m}, \\
& W(y) \geq W\left(y_{v}\right)+\left(c_{0}-v\right)\left(y-y_{v}\right) \quad \text { for all } y>y_{v},
\end{aligned}
$$

so that 2.3 yields

$$
\begin{aligned}
w^{\prime}(y) & =W(y)-k w_{+}^{m}(y)+\frac{y}{2} w(y) \\
& \geq W\left(y_{v}\right)-\left(c_{0}-v\right) y_{v}-k\left(w_{\infty}+v\right)_{+}^{m}+\left(c_{0}-v+\frac{w_{\infty}-v}{2}\right) y, \quad y>y_{v} .
\end{aligned}
$$

Since $w$ remains bounded, we therefore have $c_{0}-v+\left(w_{\infty}-v\right) / 2 \leq 0$ and thus, since $v>0$ was arbitrary, $c_{0} \leq-w_{\infty} / 2$. Using a similar argument, one can see that also $c_{0} \geq-w_{\infty} / 2$ and therefore $c_{0}=-w_{\infty} / 2$, that is, $\frac{1}{2(m-1)} w_{\infty}=\left(w_{\infty}\right)_{+}^{2 m-1}$. This yields the claim. 


\section{Comparison results}

In this section we state two useful comparison results for second-order ODEs which will frequently be applied throughout this paper.

The first tool concerns linear equations with variable coefficients and is very much in the spirit of [10, p. 123].

Lemma 3.1. Suppose that $-\infty<\xi_{0}<\xi_{\infty} \leq \infty, a, b \in C^{0}\left(\left[\xi_{0}, \xi_{\infty}\right)\right)$, and that $\varphi=$ $\varphi(\xi)$ and $\psi=\psi(\xi)$ belong to $W_{\mathrm{loc}}^{2, \infty}\left(\left[\xi_{0}, \xi_{\infty}\right)\right)$ and satisfy

$$
\left\{\begin{array}{l}
\varphi^{\prime \prime} \geq a(\xi) \varphi^{\prime}+b(\xi) \varphi \quad \text { a.e. in }\left(\xi_{0}, \xi_{\infty}\right) \\
\psi^{\prime \prime} \leq a(\xi) \psi^{\prime}+b(\xi) \psi \quad \text { a.e. in }\left(\xi_{0}, \xi_{\infty}\right) \\
\psi>0 \text { in }\left(\xi_{0}, \xi_{\infty}\right) \\
\varphi\left(\xi_{0}\right)=\psi\left(\xi_{0}\right), \quad \varphi^{\prime}\left(\xi_{0}\right) \geq \psi^{\prime}\left(\xi_{0}\right)>0 .
\end{array}\right.
$$

Then

$$
\varphi \geq \psi \quad \text { and } \quad \varphi^{\prime} \psi \geq \varphi \psi^{\prime} \quad \text { in }\left(\xi_{0}, \xi_{\infty}\right) .
$$

Proof. After a translation we may restrict ourselves to the case $\xi_{0}=0$ and set $\xi_{1}:=$ $\sup \left\{\xi \in\left(0, \xi_{\infty}\right) \mid \varphi>0\right.$ on $\left.(0, \xi)\right\} \leq \xi_{\infty}$. Since $\varphi(0) \geq 0$ and $\varphi^{\prime}(0) \geq \psi^{\prime}(0)>0$, $\xi_{1}$ is well-defined and positive. By $3.1, \rho(\xi):=\left(\varphi^{\prime} \psi-\varphi \psi^{\prime}\right)(\xi)$ satisfies

$$
\rho^{\prime}=\varphi^{\prime \prime} \psi-\varphi \psi^{\prime \prime} \geq\left(a \varphi^{\prime}+b \varphi\right) \psi-\varphi\left(a \psi^{\prime}+b \psi\right)=a \rho \quad \text { a.e. in }\left(0, \xi_{1}\right)
$$

and thus, since $\rho(0) \geq 0$, we have $\rho \geq 0$ in $\left(0, \xi_{1}\right)$. This, however, means that $\zeta:=\varphi / \psi$ is nondecreasing on $\left(0, \xi_{1}\right)$. Since (3.1) implies $\zeta(0)=1$ if $\psi(0) \neq 0$ and, by l'Hôspital's rule, $\zeta(0)=\lim _{\xi \rightarrow 0} \varphi^{\prime}(\xi) / \psi^{\prime}(\xi) \geq 1$ in case $\psi(0)=0$, we conclude that in any case $\zeta(\xi) \geq \zeta(0) \geq 1$ for all $\xi \in\left(0, \xi_{1}\right)$. Therefore $\xi_{1}=\xi_{\infty}$ and 3.2$)$ is valid.

Concerning differential inequalities with constant coefficients, we obtain a sharper result.

Lemma 3.2. Let $a$ and $b$ be real numbers satisfying

$$
a^{2} / 4+b>0 .
$$

Suppose that $-\infty<\xi_{0}<\xi_{\infty} \leq \infty$, and that $\varphi \in W_{\mathrm{loc}}^{2, \infty}\left(\left[\xi_{0}, \xi_{\infty}\right)\right)$ satisfies

$$
\varphi^{\prime \prime} \geq a \varphi^{\prime}+b \varphi \quad \text { a.e. in }\left(\xi_{0}, \xi_{\infty}\right) \text {. }
$$

Then

$$
\begin{aligned}
\varphi(\xi) \geq & \frac{\varphi^{\prime}\left(\xi_{0}\right)-\lambda^{-} \varphi\left(\xi_{0}\right)}{\lambda^{+}-\lambda^{-}} e^{\lambda^{+}\left(\xi-\xi_{0}\right)} \\
& +\frac{\lambda^{+} \varphi\left(\xi_{0}\right)-\varphi^{\prime}\left(\xi_{0}\right)}{\lambda^{+}-\lambda^{-}} e^{\lambda^{-}\left(\xi-\xi_{0}\right)}, \quad \xi \in\left(\xi_{0}, \xi_{\infty}\right),
\end{aligned}
$$

with

$$
\lambda^{ \pm}=a / 2 \pm \sqrt{a^{2} / 4+b}
$$


Moreover,

$$
\begin{aligned}
& \text { if } \varphi \geq 0 \text { on }\left(\xi_{0}, \xi_{\infty}\right) \text { and } \varphi^{\prime}\left(\xi_{0}\right) \geq \lambda \varphi\left(\xi_{0}\right) \text { for some } \lambda \in\left[\lambda^{-}, \lambda^{+}\right] \\
& \text {then } \varphi^{\prime} \geq \lambda \varphi \text { for all } \xi \in\left(\xi_{0}, \xi_{\infty}\right) .
\end{aligned}
$$

Proof. We may again assume that $\xi_{0}=0$. We substitute $\varphi(\xi)=e^{\lambda \xi} \rho(\xi)$ with $\lambda \in \mathbb{R}$ and calculate

$$
\begin{aligned}
\varphi^{\prime} & =e^{\lambda \xi}\left(\rho^{\prime}+\lambda \rho\right), \\
\varphi^{\prime \prime} & =e^{\lambda \xi}\left(\rho^{\prime \prime}+2 \lambda \rho^{\prime}+\lambda^{2} \rho\right) .
\end{aligned}
$$

Therefore 3.4 transforms into

$$
\begin{aligned}
\rho^{\prime \prime} & \geq-\left(2 \lambda \rho^{\prime}+\lambda^{2} \rho\right)+a\left(\rho^{\prime}+\lambda \rho\right)+b \rho \\
& =(a-2 \lambda) \rho^{\prime}-\left(\lambda^{2}-a \lambda-b\right) \rho \quad \text { a.e. in }\left(0, \xi_{\infty}\right) .
\end{aligned}
$$

Choosing $\lambda=\lambda^{+}$here, we see that $\rho^{\prime \prime} \geq-\left(\lambda^{+}-\lambda^{-}\right) \rho^{\prime}$ a.e. in $\left(0, \xi_{\infty}\right)$. Integrating this inequality we have $\rho^{\prime}(\xi) \geq e^{-\left(\lambda^{+}-\lambda^{-}\right) \xi} \rho^{\prime}(0)$ for all $\xi \in\left(0, \xi_{\infty}\right)$. One further integration yields

$$
\begin{aligned}
\varphi(\xi) & =e^{\lambda^{+} \xi} \rho(\xi) \\
& \geq e^{\lambda^{+} \xi}\left[\rho(0)-\frac{1}{\lambda^{+}-\lambda^{-}}\left(e^{-\left(\lambda^{+}-\lambda^{-}\right) \xi}-1\right) \rho^{\prime}(0)\right] \\
& =e^{\lambda^{+} \xi} \varphi(0)-\frac{1}{\lambda^{+}-\lambda^{-}}\left(e^{\lambda^{-} \xi}-e^{\lambda^{+} \xi}\right)\left(\varphi^{\prime}(0)-\lambda^{+} \varphi(0)\right)
\end{aligned}
$$

and thereby proves 3.5 .

To see the implication 3.7 , we note that $\lambda \in\left[\lambda^{-}, \lambda^{+}\right]$is equivalent to $\lambda^{2}-a \lambda-b \leq 0$, so that the hypothesis $\varphi \geq 0$ yields $\rho^{\prime \prime} \geq(a-2 \lambda) \rho^{\prime}$ in $\left(0, \xi_{\infty}\right)$ by 3.8). Therefore $\rho^{\prime}(\xi)=e^{-\lambda \xi}\left(\varphi^{\prime}(\xi)-\lambda \varphi(\xi)\right)$ preserves its initial nonnegativity for all $\xi \in\left(0, \xi_{\infty}\right)$.

\section{Solutions without local minima}

The goal of the present section is to prove that if $\sigma>0$ is sufficiently small then the solution $w_{\sigma}$ of (2.1), 2.2 will increase for small $y$, attain a local maximum and then decrease to $-\infty$, so that in particular it will not attain a local minimum in $(0, \infty)$. In order to prepare the proof of this statement (Theorem 4.3), let us demonstrate two general features of solutions of 2.1.

The first of them means, roughly speaking, that if a solution $w$ of 2.1 crosses $\mu$ 'from above' at some $y_{1}$ large enough then it must decrease for $y>y_{1}$ and eventually become negative, regardless of the size of $w^{\prime}\left(y_{1}\right)$.

Lemma 4.1. Assume that $w$ is a solution of 2.1. If $w\left(y_{1}\right)=\mu$ and $w^{\prime}\left(y_{1}\right)<0$ occurs for some $y_{1} \geq 6+2 k m \mu^{m-1}$ then $w^{\prime}(y)<0$ for all $y \geq y_{1}$ and $w(y) \rightarrow-\infty$ as $y \rightarrow \infty$. 
Proof. Let

$$
y_{2}:=\sup \left\{y>y_{1} \mid w>0 \text { and } w^{\prime}<0 \text { in }\left(y_{1}, y\right)\right\} \leq \infty .
$$

Then on $\left(y_{1}, y_{2}\right)$ we have $w<\mu$ and thus

$$
w^{\prime \prime} \leq \frac{y_{1}}{2} w^{\prime}-k m \mu^{m-1} w^{\prime}+\frac{1}{2(m-1)} w-w^{2 m-1} .
$$

Let $\varphi(y):=\mu-w(y)$. Then using the convexity inequality $(1-\xi)^{2 m-1} \geq 1-(2 m-1) \xi$ for $0 \leq \xi<1$ as well as the properties of $y_{1}$ and $\mu$, we can estimate

$$
\begin{aligned}
\varphi^{\prime \prime} & \geq\left(\frac{y_{1}}{2}-k m \mu^{m-1}\right) \varphi^{\prime}-\frac{1}{2(m-1)}(\mu-\varphi)+(\mu-\varphi)^{2 m-1} \\
& \geq 4 \varphi^{\prime}-\frac{1}{2(m-1)}(\mu-\varphi)+\mu^{2 m-1}+(2 m-1) \mu^{2 m-2} \varphi \\
& =4 \varphi^{\prime}-\varphi \quad \text { in }\left(y_{1}, y_{2}\right) .
\end{aligned}
$$

Since $4^{2} / 4-1=3>0$ and $\varphi>0$ in $\left(y_{1}, y_{2}\right)$, Lemma 3.2 shows that $\varphi^{\prime} \geq 2 \varphi$ in $\left(y_{1}, y_{2}\right)$, because $\varphi^{\prime}\left(y_{1}\right)-2 \varphi\left(y_{1}\right)>0$ and $2 \in\left[\lambda^{-}, \lambda^{+}\right]$with $\lambda^{ \pm}=2 \pm \sqrt{3}$ as in 3.6 . Therefore $y_{2}$ must be finite, since $\varphi$ grows exponentially in $\left(y_{1}, y_{2}\right)$, and furthermore $w\left(y_{2}\right)=0$ and $w^{\prime}\left(y_{2}\right)<0$. But due to Lemma 2.1. $w$ cannot have local minima as long as $w<0$, whence $w$ actually decreases throughout $\left(y_{1}, \infty\right)$. Now (2.1) shows that $w$ is even concave on $\left(y_{2}, \infty\right)$ and therefore approaches $-\infty$ as $y \rightarrow \infty$.

The second lemma asserts that all solutions of 2.1) eventually decrease.

Lemma 4.2. Let $w$ be a solution of 2.1]. Then there exists $y_{1}>0$ such that $w^{\prime}(y) \leq 0$ for all $y>y_{1}$.

Proof. Assume that the lemma is false. Then either $w^{\prime}(y)>0$ for all large $y$, or both $\left\{y>0 \mid w^{\prime}(y)>0\right\}$ and $\left\{y>0 \mid w^{\prime}(y)<0\right\}$ are unbounded. In the latter case, $w$ must have unbounded sequences of local minima and maxima. Since, due to Lemma 2.1. these minima have their ordinates in $(0, \mu)$ and the maxima lie above $\mu$, this means that there exists a sequence of numbers $y_{j} \rightarrow \infty$ such that $w\left(y_{j}\right)=\mu$ and $w^{\prime}\left(y_{j}\right)<0$, contradicting Lemma 4.1 .

Therefore we are left with the case that $w^{\prime}(y)>0$ for large $y$; then $w(y) \nearrow w_{\infty}$ as $y \rightarrow \infty$ for some $w_{\infty} \leq \infty$. In view of Lemma 2.2 we have either $w_{\infty}=0$ or $w_{\infty}=\mu$ or $w_{\infty}=\infty$. In the first two cases, $w(y) \leq \mu$ for large $y$, so that from 2.1] we obtain

$$
w^{\prime \prime}(y) \geq\left(y / 2-k m \mu^{m-1}\right) w^{\prime}(y) \quad \text { for large } y,
$$

which is impossible for bounded $w$. We thus only need to exclude that $w(y) \nearrow \infty$ as $y \rightarrow \infty$. 
To do this, we first integrate 2.1$)$ over $(0, y)$ to obtain

$$
\begin{aligned}
w^{\prime}(y)-w^{\prime}(0)= & \int_{0}^{y} \frac{\xi}{2} w^{\prime}(\xi) d \xi-k w_{+}^{m}(y)+\frac{1}{2(m-1)} \int_{0}^{y} w(\xi) d \xi-\int_{0}^{y} w_{+}^{2 m-1}(\xi) d \xi \\
= & \frac{y}{2} w(y)-k w_{+}^{m}(y)+\frac{2-m}{2(m-1)} \int_{0}^{y} w(\xi) d \xi \\
& -\int_{0}^{y} w_{+}^{2 m-1}(\xi) d \xi \quad \text { for all } y>0
\end{aligned}
$$

In order to show the dominance of the term involving $w_{+}^{2 m-1}$ in 4.1 in the case under consideration, we first claim that there exists $y_{0}>0$ such that

$$
\frac{2-m}{2(m-1)} \int_{0}^{y} w(\xi) d \xi \leq \frac{1}{4} \int_{0}^{y} w_{+}^{2 m-1}(\xi) d \xi, \quad y \geq y_{0} .
$$

Indeed, this easily follows from the observation that since $w(y) \rightarrow \infty$, the function $\zeta(y):=\frac{1}{4} w_{+}^{2 m-1}(y)-\frac{2-m}{2(m-1)} w(y)$ tends to $\infty$ as $y \rightarrow \infty$, which clearly implies $\int_{0}^{y} \zeta(\xi) d \xi \rightarrow \infty$ as $y \rightarrow \infty$.

Next, we claim that there is a sequence of numbers $\bar{y}_{j} \rightarrow \infty$ such that

$$
\frac{\bar{y}_{j}}{2} w\left(\bar{y}_{j}\right) \leq \frac{1}{4} \int_{0}^{\bar{y}_{j}} w_{+}^{2 m-1}(\xi) d \xi, \quad j \in \mathbb{N} .
$$

In fact, suppose 4.3 is false. Then there exists $\bar{y}>0$ such that

$$
y w(y)>\frac{1}{2} \int_{0}^{y} w_{+}^{2 m-1}(\xi) d \xi, \quad y \geq \bar{y} .
$$

Since $\frac{1}{y} \int_{0}^{y} w_{+}^{2 m-1}(\xi) d \xi \rightarrow \infty$ as $y \rightarrow \infty$, we may assume $\bar{y}$ to be so large that

$$
C:=\frac{1}{2} \int_{0}^{\bar{y}} w_{+}^{2 m-1}(\xi) d \xi>2^{1 / 2(m-1)} \bar{y} .
$$

Let $v(y):=y w(y)$ for $y \geq \bar{y}$. Then, by (4.4),

$$
\begin{aligned}
v(y) & >\frac{1}{2} \int_{0}^{y} w_{+}^{2 m-1}(\xi) d \xi=\frac{1}{2} \int_{0}^{\bar{y}} w_{+}^{2 m-1}(\xi) d \xi+\int_{\bar{y}}^{y}\left(\frac{v(\xi)}{\xi}\right)^{2 m-1} d \xi \\
& =C+\frac{1}{2} \int_{\bar{y}}^{y}\left(\frac{v(\xi)}{\xi}\right)^{2 m-1} d \xi, \quad y \geq \bar{y} ;
\end{aligned}
$$

in particular, $v(\bar{y})>C$. Let $\psi$ denote the solution of $\psi^{\prime}(y)=\frac{1}{2}(\psi(y) / y)^{2 m-1}$ for $y>\bar{y}$ with $\psi(\bar{y})=C$, which is explicitly given by

$$
\psi(y)=\left(C^{2-2 m}-\frac{1}{2} \bar{y}^{2-2 m}+\frac{1}{2} y^{2-2 m}\right)^{-1 / 2(m-1)} .
$$


Since $C^{2-2 m}-\frac{1}{2} \bar{y}^{2-2 m}<0$ due to 4.5 , we have

$$
\psi(y) \rightarrow \infty \quad \text { as } y \rightarrow \hat{y}:=\left(\bar{y}^{2-2 m}-2 C^{2-2 m}\right)^{-1 / 2(m-1)} \in(\bar{y}, \infty) .
$$

But $\psi(y)=C+\int_{\bar{y}}^{y}(\psi(\xi) / \xi)^{2 m-1} d \xi$ for all $y \geq \bar{y}$, whence $v>\psi$ in $[\bar{y}, \hat{y})$ by a standard comparison argument. Consequently, $w$ must blow up at some finite $y$. This contradiction establishes (4.3).

Using (4.2) and 4.3 in 4.1, we finally obtain, for large $j \in \mathbb{N}$,

$$
\begin{aligned}
-w^{\prime}(0) & \leq w^{\prime}\left(\bar{y}_{j}\right)-w^{\prime}(0) \leq-\frac{1}{2} \int_{0}^{y_{j}} w_{+}^{2 m-1}(\xi) d \xi \\
& \rightarrow-\infty \quad \text { as } j \rightarrow \infty,
\end{aligned}
$$

which is absurd and thereby proves the lemma.

Now the main result of this section is an easy consequence of the above preparations and the continuous dependence of solutions of 2.1, 2.2) on $\sigma$.

Theorem 4.3. There is a positive number $\sigma_{0}$ such that for all $\sigma \in\left(0, \sigma_{0}\right)$, the function $w_{\sigma}$ has precisely one local maximum and no local minima in $(0, \infty)$.

Proof. Let $\bar{y}:=6+2 k m \mu^{m-1}$ denote the number from Lemma 4.1. Since the mapping $\sigma \mapsto w_{\sigma}$ is continuous from $[0, \infty)$ to $C^{0}([0, \bar{y}])$, there is $\sigma_{0}>0$ such that $w_{\sigma}(y) \leq \frac{1}{2} \mu$ in $[0, \bar{y}]$ for all $\sigma<\sigma_{0}$. For any such $\sigma, w_{\sigma}^{\prime}$ must have a minimal zero $y_{1}>0$ due to Lemma 4.2, which necessarily corresponds to a local maximum. By (2.1), $w_{\sigma}\left(y_{1}\right)>\mu$ and hence $y_{1}>\bar{y}$. Now if $w_{\sigma}^{\prime}$ had another zero in $\left(y_{1}, \infty\right)$ then $w_{\sigma}$ should have a positive local minimum at some $y_{2}>y_{1}$ with $w_{\sigma}\left(y_{2}\right)<\mu$ in virtue of Lemma 2.1. Thus, there would be $y_{3} \in\left(y_{1}, y_{2}\right)$ such that $w_{\sigma}\left(y_{3}\right)=\mu$ and $w_{\sigma}^{\prime}\left(y_{3}\right)<0$. But since $y_{3}>y_{1}>\bar{y}$, Lemma 4.1 then provides a contradiction and thereby proves the theorem.

\section{Solutions attaining a local minimum}

In this section we shall see that if $k$ is sufficiently large then for some $\sigma>0$, the solution of 2.1, 2.2 attains a positive local minimum somewhere in $(0, \infty)$. Our approach towards this crucial tool for the proof of Theorem 1.1 is by applying a twosided shooting method, with the origin of shooting located at a point $\left(y_{0}, w_{s}^{+}\left(y_{0}\right)\right)$ with some appropriate $y_{0}>0$. Here the essential ingredient will be an instability property of $w_{s}^{+}$, according to which backward shooting from $\left(y_{0}, w_{s}^{+}\left(y_{0}\right)\right)$ with any initial slope $w^{\prime}\left(y_{0}\right)>\left(w_{s}^{+}\right)^{\prime}\left(y_{0}\right)$ will force $w$ to have a zero in $(0, \infty)$, no matter how small the deviation $w^{\prime}\left(y_{0}\right)-\left(w_{s}^{+}\right)^{\prime}\left(y_{0}\right)$ is. This freedom in the choice of $w^{\prime}\left(y_{0}\right)$ will be used in a second step: Roughly speaking, shooting forward from $\left(y_{0}, w_{s}^{+}\left(y_{0}\right)\right)$ with $w^{\prime}\left(y_{0}\right)-\left(w_{s}^{+}\right)^{\prime}\left(y_{0}\right)$ sufficiently small but positive will produce a solution $w$ which on one hand, by continuous dependence, remains close to $w_{s}^{+}$in a sufficiently large interval—so that, in particular, it enters the region $w<\mu$ where local minima occur; on the other hand, however, the term 
$(y / 2) w^{\prime}$ on the right of 2.1 gives rise to a further instability feature of $w_{s}^{+}$, which entails that any such $w$ exhibits a tendency to behave like $e^{y^{2} / 4}$ intermediately and therefore will eventually be repelled from $w_{s}^{+}$until it leaves the region $w<\mu$. As a consequence, $w$ must have a local minimum. Finally, glueing together the backward and forward shooting products and applying a further continuous dependence argument, we will end up with a solution of 2.1], (2.2) that in fact has a local minimum (Theorem 5.6.

To carry out this plan it will be convenient to rescale solutions $w$ of 2.1 in the following way:

$$
w(y)=f(y) w_{s}^{+}(y), \quad y>0 .
$$

Recalling the explicit representation $w_{s}^{+}(y)=A y^{-1 /(m-1)}$, we compute that $f$ satisfies

$$
f^{\prime \prime}=\frac{y}{2} f^{\prime}+\frac{2}{(m-1) y} f^{\prime}-\frac{k m A^{m-1}}{y} f_{+}^{m-1} f^{\prime}+\frac{1}{y^{2}} \Phi(f), \quad y>0,
$$

with

$$
\Phi(s)=-\frac{m}{(m-1)^{2}} s+\frac{k m A^{m-1}}{m-1} s_{+}^{m}-A^{2 m-2} s_{+}^{2 m-1}, \quad s \in \mathbb{R} .
$$

Now given $y_{0}>0$, we rescale the interval $\left(0, y_{0}\right)$ by setting $z:=-\ln \left(y / y_{0}\right)$ for $y \in$ $\left(0, y_{0}\right)$, so that the backward function

$$
g_{b}(z) \equiv g_{b}\left(z ; y_{0}\right):=f\left(y_{0} e^{-z}\right), \quad z \in(0, \infty)
$$

is a solution of

$$
g_{b}^{\prime \prime}=-\left(\frac{m+1}{m-1}+\frac{y_{0}^{2}}{2} e^{-2 z}\right) g_{b}^{\prime}+k m A^{m-1} g_{b+}^{m-1} g_{b}^{\prime}+\Phi\left(g_{b}\right), \quad z \in(0, \infty) .
$$

Similarly, the forward transformation $z=\ln \left(y / y_{0}\right), y \in\left(y_{0}, \infty\right)$, and

$$
g_{f}(z) \equiv g_{f}\left(z ; y_{0}\right):=f\left(y_{0} e^{z}\right), \quad z \in(0, \infty),
$$

lead to the equation

$$
g_{f}^{\prime \prime}=\left(\frac{m+1}{m-1}+\frac{y_{0}^{2}}{2} e^{2 z}\right) g_{f}^{\prime}-k m A^{m-1} g_{f+}^{m-1} g_{f}^{\prime}+\Phi\left(g_{f}\right), \quad z \in(0, \infty) .
$$

Lemma 5.1. The function $\Phi$ is concave on $[1, \infty)$ and

$$
\Phi(s) \leq\left(k m A^{m-1}-\frac{2 m}{m-1}\right)(1-s) \quad \text { for all } s \in(0, \infty) .
$$


Proof. From

$$
\Phi^{\prime}(s)=-\frac{m}{(m-1)^{2}}+\frac{k m^{2} A^{m-1}}{m-1} s^{m-1}-(2 m-1) A^{2 m-2} s^{2 m-2}, \quad s>0,
$$

and the fact that $\Phi(1)=-\frac{m}{(m-1)^{2}}+\frac{k m A^{m-1}}{m-1}-A^{2 m-2}=0$ by 2.5 , it follows that

$$
\Phi^{\prime}(1)=\frac{2 m}{m-1}-k m A^{m-1},
$$

and that $\Phi^{\prime \prime}$ has precisely one zero $s_{0}$ in $(0, \infty)$. Since $k m A^{m-1}>2 m /(m-1)$ whenever $k>2 / \sqrt{m}$, we thus find that $\Phi^{\prime}(1)<0$; as also $\Phi^{\prime}(0)<0$, we conclude that $\Phi^{\prime}$ has two zeros in $(0,1)$, say at $0<s_{1}<s_{2}<1$. Therefore we necessarily have $s_{0} \in\left(s_{1}, s_{2}\right)$ and in particular $\Phi^{\prime \prime}(s)<0$ for all $s \geq s_{2}$. This proves the concavity of $\Phi$ on $[1, \infty)$ and, moreover, that $\Phi^{\prime}(s) \geq \Phi^{\prime}(1)$ for all $s \in\left(s_{1}, 1\right)$ and $\Phi^{\prime}(s) \leq \Phi^{\prime}(1)$ for each $s \geq 1$, whence $\Phi(s)=\Phi(1)+\int_{1}^{s} \Phi^{\prime}(\xi) d \xi \leq \Phi^{\prime}(1)(s-1)$ for all $s>s_{1}$. Therefore 5.6 yields [5.5), because $\Phi(s) \leq 0$ for $s \in\left(0, s_{1}\right]$.

\subsection{Shooting backwards}

The following auxiliary lemma is an application of Lemma 3.1 to a particular differential inequality that will arise in the backward shooting procedure in Lemma 5.3 below.

Lemma 5.2. There exists $M_{0}>0$ such that if $z_{\infty}>0$ and $\varphi \in C^{2}\left(\left[0, z_{\infty}\right)\right)$ is a function satisfying

$$
\left\{\begin{array}{l}
\varphi^{\prime \prime} \geq M\left(1-e^{-2 z}\right) \varphi^{\prime}-N \varphi \quad \text { in }\left(0, z_{\infty}\right) \\
\varphi(0)=0, \quad \varphi^{\prime}(0)=1
\end{array}\right.
$$

with some $M \geq M_{0}$ and $N \in[0, M]$, then

$$
\varphi(z) \geq \begin{cases}z & \text { if } 0 \leq z<\sqrt{3 / M}, \\ \frac{\sqrt{3}}{e \sqrt{M}} e^{\sqrt{M / 3} z} & \text { if } \sqrt{3 / M} \leq z<z_{\infty},\end{cases}
$$

and

$$
\varphi^{\prime}(z) \geq \begin{cases}\frac{1}{z} \varphi(z) & \text { if } 0<z<\sqrt{3 / M} \\ \sqrt{M / 3} \varphi(z) & \text { if } \sqrt{3 / M} \leq z<z_{\infty} .\end{cases}
$$

Proof. Since $\frac{d}{d s}(-\ln (1-s))=\frac{1}{1-s} \rightarrow 1$ as $s \rightarrow 0$, it is possible to choose $v_{0} \in$ $(0,(2 \ln 2) / 3)$ small such that $-\ln (1-v) \leq 3 v / 2$ for all $v \in\left[0, v_{0}\right]$. We set $M_{0}:=$ $16 / 3 v_{0}^{2}$ and, given $M \geq M_{0}$, let

$$
\beta=\beta(M):=\sqrt{M / 3}, \quad v=v(M):=4 / \sqrt{3 M}, \quad z_{0}=z_{0}(M):=1 / \beta(M) .
$$


Then $v(M) \leq v_{0}$ and

$$
-\frac{\ln (1-v(M))}{2} \leq z_{0}(M) \leq \frac{\ln 2}{2} \quad \text { for all } M \geq M_{0},
$$

because $v \leq v_{0}<(2 \ln 2) / 3$ implies $z_{0}(m)+\sqrt{3 / M} \leq \sqrt{3 / M_{0}}=3 v_{0} / 4<(\ln 2) / 2$ and, on the other hand, $-\ln (1-v) / 2 \leq 3 v / 4=\sqrt{3 / M}=z_{0}(M)$.

We now define

$$
\psi(z):= \begin{cases}z, & 0 \leq z<z_{0}(M), \\ \frac{1}{\beta(M)} e^{\beta(M) \cdot\left(z-z_{0}(M)\right)} \equiv \frac{1}{e \beta(M)} e^{\beta(M) z}, & z \geq z_{0}(M) .\end{cases}
$$

Then both $\psi$ and $\psi^{\prime}$ are continuous at $z=z_{0}(M)$, so that $\psi \in W_{\text {loc }}^{2, \infty}([0, \infty))$. Moreover, $\psi(0)=0$ and $\psi^{\prime}(0)=1$, and since it can easily be checked that $1-e^{-2 z} \geq z$ for all $z \in[0,(\ln 2) / 2]$, we have

$$
\begin{aligned}
\psi^{\prime \prime}-M\left(1-e^{-2 z}\right) \psi^{\prime}+N \psi & =-M\left(1-e^{-2 z}\right)+N z \leq-M z+N z \\
& \leq 0 \quad \text { in }\left(0, z_{0}(M)\right)
\end{aligned}
$$

due to the second inequality in 5.10 and the assumption $N \leq M$.

Whenever $z \geq z_{0}(M)$, however, the first inequality in $(5.10)$ ensures that

$$
1-e^{-2 z} \geq 1-e^{-2 z_{0}(M)} \geq 1-e^{-2 \cdot(-\ln (1-v(M))) / 2}=v(M),
$$

so that in this case we obtain

$$
\begin{aligned}
\psi^{\prime \prime}-M\left(1-e^{-2 z}\right) \psi^{\prime}+N \psi & =\frac{1}{\beta} e^{\beta\left(z-z_{0}\right)}\left[\beta^{2}-M\left(1-e^{-2 z}\right) \beta+N\right] \\
& \leq \frac{1}{\beta} e^{\beta\left(z-z_{0}\right)}\left[\beta^{2}-v M \beta+M\right] \\
& \leq \frac{1}{\beta} e^{\beta\left(z-z_{0}\right)}\left[\frac{M}{3}-\frac{v}{\sqrt{3}} M \sqrt{M}+M\right] \\
& \leq \frac{1}{\beta} e^{\beta\left(z-z_{0}\right)} \frac{M}{3}(4-v \sqrt{3 M}) \\
& =0 \quad \text { in }\left(z_{0}(M), \infty\right)
\end{aligned}
$$

in view of our choice of $v=v(M)$. Combined with (5.11), this allows us to conclude with the aid of the comparison in Lemma 3.1 that $\varphi \geq \psi$ and $\varphi^{\prime} \psi \geq \varphi \psi^{\prime}$ in $\left[0, z_{\infty}\right)$. Since $\psi^{\prime} / \psi \equiv \beta(M)=\sqrt{M / 3}$ for $z>z_{0}$, this yields $[5.8$ and 5.9].

With this preparation at hand, we can now prove that shooting backwards in fact produces solutions with zeros, provided that the shooting origin is chosen appropriately and, which might not be surprising, if the convection term in 2.1) is strong enough. 
Lemma 5.3. Let $m>1$. Then there exists $k_{0}>2 / \sqrt{m}$ with the property that for any $k \geq k_{0}$ one can find $\varepsilon_{0}$ such that for all $\varepsilon \in\left(0, \varepsilon_{0}\right)$, the solution $g_{b}=g_{b}\left(\cdot ; y_{0}\right)$ of the backward shooting problem

$$
\left\{\begin{array}{l}
g_{b}^{\prime \prime}=-\left(\frac{m+1}{m-1}+\frac{y_{0}^{2}}{2} e^{-2 z}\right) g_{b}^{\prime}+k m A^{m-1} g_{b+}^{m-1} g_{b}^{\prime}+\Phi\left(g_{b}\right), \quad z>0 \\
g_{b}(0)=1, \quad g_{b}^{\prime}(0)=-\varepsilon
\end{array}\right.
$$

has a zero in $(0, \infty)$ if we choose

$$
y_{0}:=\sqrt{2} \sqrt{k m A^{m-1}-\frac{2 m}{m-1}} .
$$

Remark. As already mentioned in Lemma 5.1, $k m A^{m-1}>2 m /(m-1)$ for any $k>$ $2 / \sqrt{m}$, so that $y_{0}$ is well defined.

Proof. For $k \geq 2 / \sqrt{m}$ we abbreviate

$$
B \equiv B(k):=k m A^{m-1} \equiv \frac{k^{2} m^{2}+k m \sqrt{k^{2} m^{2}-4 m}}{2(m-1)}
$$

and observe that restricting $k \geq k_{0}$ is equivalent to saying $B \geq B\left(k_{0}\right)$.

Writing $g$ instead of $g_{b}$ throughout and setting $G:=1-g$, we see from Lemma 5.1 that

$$
\begin{aligned}
G^{\prime \prime}= & -\left(\frac{m+1}{m-1}+\frac{y_{0}^{2}}{2} e^{-2 z}\right) G^{\prime}+B g^{m-1} G^{\prime}-\Phi(g) \\
\geq & -\left(\frac{m+1}{m-1}+\frac{y_{0}^{2}}{2} e^{-2 z}\right) G^{\prime}+B g^{m-1} G^{\prime} \\
& -\left(B-\frac{2 m}{m-1}\right) G \text { in }\left(0, z_{\infty}\right),
\end{aligned}
$$

where $z_{\infty}:=\sup \{z>0 \mid g>0$ in $(0, z)\} \leq \infty$. Since $z_{\infty}<\infty$ would mean that $g$ has a zero, in order to prove the lemma it is sufficient to assume henceforth that $z_{\infty}=\infty$ and show that this is impossible if $B$ is appropriately large and $\varepsilon$ suitably small.

To this end, let us set

$$
z_{1} \equiv z_{1}(B, \varepsilon):=\sup \left\{z>0 \mid 1-1 / B \leq g^{m-1} \leq 1 \text { and } G^{\prime} \geq 0 \text { on }(0, z)\right\} \leq \infty
$$

for $B>B_{1}:=\max \{1, B(2 / \sqrt{m})\}$ and $\varepsilon>0$. Then $g(0)=1$ and $g^{\prime}(0)<0$ guarantee that $z_{1}>0$, and 5.13 says that

$$
\begin{aligned}
G^{\prime \prime} & \geq-\left(\frac{m+1}{m-1}+\frac{y_{0}^{2}}{2} e^{-2 z}\right) G^{\prime}+B\left(1-\frac{1}{B}\right) G^{\prime}-\left(B-\frac{2 m}{m-1}\right) G \\
& =\left(B-\frac{2 m}{m-1}+\frac{y_{0}^{2}}{2} e^{-2 z}\right) G^{\prime}-\left(B-\frac{2 m}{m-1}\right) G \\
& =\left(B-\frac{2 m}{m-1}\right)\left(1-e^{-2 z}\right) G^{\prime}-\left(B-\frac{2 m}{m-1}\right) G \quad \text { in }\left(0, z_{1}\right),
\end{aligned}
$$


since $y_{0}^{2} / 2=B-2 m /(m-1)$. We now apply Lemma 5.2 to $\varphi:=G / \varepsilon$ to obtain

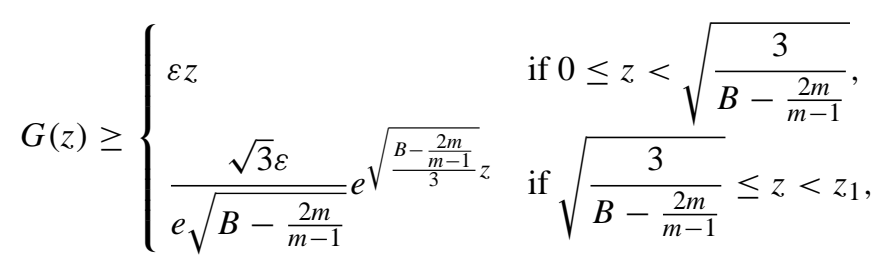

and

$$
G^{\prime}(z) \geq \begin{cases}\frac{1}{z} G(z) & \text { if } 0 \leq z<\sqrt{\frac{3}{B-\frac{2 m}{m-1}}}, \\ \sqrt{\frac{B-\frac{2 m}{m-1}}{3}} G(z) & \text { if } \sqrt{\frac{3}{B-\frac{2 m}{m-1}}} \leq z<z_{1} .\end{cases}
$$

In particular, this implies that $z_{1}=z_{1}(B, \varepsilon)$ is finite for all $B>B_{1}$ and $\varepsilon>0$, and that $G^{\prime}\left(z_{1}\right)>0$ and hence $g^{m-1}\left(z_{1}\right)=1-1 / B$. Since the solution of 5.12) depends continuously on $\varepsilon$ in $C^{0}\left(\left[0, \bar{z}_{\infty}\right]\right)$ for all $\bar{z}_{\infty} \in(0, \infty)$, we therefore can, given any $B>B_{1}$, pick some $\varepsilon_{0}=\varepsilon_{0}(B)>0$ in such a way that

$$
z_{1}(B, \varepsilon)>\sqrt{\frac{3}{B-\frac{2 m}{m-1}}}, \quad \varepsilon<\varepsilon_{0}(B),
$$

and, for later purpose, that

$$
z_{1}(B, \varepsilon) \geq-\frac{1}{2} \ln \frac{2}{y_{0}^{2}} \equiv \frac{1}{2} \ln \left(B-\frac{2 m}{m-1}\right), \quad \varepsilon<\varepsilon_{0}(B) .
$$

From 5.14) and 5.15) we thus infer that if

$$
B>B_{2}:=\max \left\{B_{1}, \frac{8 m}{m-1}\right\}
$$

and $\varepsilon<\varepsilon_{0}(B)$ then $G\left(z_{1}\right)>0$ and

$$
G^{\prime}\left(z_{1}\right) \geq \sqrt{\frac{B-\frac{2 m}{m-1}}{3}} G\left(z_{1}\right) \geq \frac{\sqrt{B}}{2} G\left(z_{1}\right) .
$$

Next, we assume that

$$
B>B_{3}:=\max \left\{B_{2},(4+\sqrt{17})^{2}\right\},
$$

so that $8 / \sqrt{B}<1-1 / B$ and hence

$$
z_{2} \equiv z_{2}(B, \varepsilon):=\sup \left\{z>z_{1} \mid 8 / \sqrt{B} \leq g^{m-1} \leq 1-1 / B \text { and } G^{\prime} \geq 0 \text { on }\left(z_{1}, z\right)\right\} \leq \infty
$$

is well-defined and larger than $z_{1}$, because $g^{m-1}\left(z_{1}\right)=1-1 / B$ and $G^{\prime}\left(z_{1}\right)>0$ due to (5.17). 
Beyond $z=z_{1}$, we have $\frac{y_{0}^{2}}{2} e^{-2 z}<1$ by 5.16 , so that

$$
\begin{aligned}
G^{\prime \prime} & =-\left(\frac{m+1}{m-1}+\frac{y_{0}^{2}}{2} e^{-2 z}\right) G^{\prime}+B g^{m-1} G^{\prime}-\Phi(g) \\
& >-\frac{2 m}{m-1} G^{\prime}+B \frac{8}{\sqrt{B}} G^{\prime}-\left(B-\frac{2 m}{m-1}\right) G \geq\left(8 \sqrt{B}-\frac{2 m}{m-1}\right) G^{\prime}-B G \\
& \geq 4 \sqrt{B} G^{\prime}-B G \quad \text { for } z \in\left(z_{1}, z_{2}\right),
\end{aligned}
$$

provided that

$$
B>B_{4}:=\max \left\{B_{3}, \frac{m^{2}}{4(m-1)^{2}}\right\} .
$$

Now Lemma 3.2 ensures that with $\lambda^{ \pm}:=2 \sqrt{B} \pm \sqrt{(2 \sqrt{B})^{2}-B}=(2 \pm \sqrt{3}) \sqrt{B}$ we have

$$
\begin{aligned}
G(z) \geq & \frac{G^{\prime}\left(z_{1}\right)-\lambda^{-} G\left(z_{1}\right)}{\lambda^{+}-\lambda^{-}} e^{\lambda^{+}\left(z-z_{1}\right)} \\
& +\frac{\lambda^{+} G\left(z_{1}\right)-G^{\prime}\left(z_{1}\right)}{\lambda^{+}-\lambda^{-}} e^{\lambda^{-}\left(z-z_{1}\right)}, \quad z \in\left(z_{1}, z_{2}\right) .
\end{aligned}
$$

Since $\lambda^{-}<\sqrt{B} / 2<\lambda^{+}$, we deduce from 5.17 that Lemma 3.2 furthermore guarantees

$$
G^{\prime}(z) \geq \frac{\sqrt{B}}{2} G(z) \quad \text { for all } z \in\left(z_{1}, z_{2}\right),
$$

and that $G^{\prime}\left(z_{1}\right)-\lambda^{-} G\left(z_{1}\right)>0$. The latter inequality shows that the right-hand side of (5.18) tends to $\infty$ as $z \rightarrow \infty$, whence $z_{2}(B, \varepsilon)$ must be finite whenever $B>B_{4}$ and $\varepsilon<\varepsilon_{0}(B)$. Moreover, $G^{\prime}\left(z_{2}\right)>0$ by 5.19 and thus $g^{m-1}\left(z_{2}\right)=8 / \sqrt{B}$. Hence, if additionally

$$
B>B_{5}:=\max \left\{B_{4}, 2^{2(m+2)}\right\}
$$

then 5.19$)$ implies

$$
G^{\prime}\left(z_{2}\right) \geq \frac{\sqrt{B}}{2}\left(1-g\left(z_{2}\right)\right)=\frac{\sqrt{B}}{2}\left(1-\left(\frac{8}{\sqrt{B}}\right)^{1 /(m-1)}\right) \geq \frac{\sqrt{B}}{4} .
$$

To initiate the final step, for $B>B_{5}$ and $\varepsilon<\varepsilon_{0}(B)$ we set

$$
z_{3} \equiv z_{3}(B, \varepsilon):=\sup \left\{z>z_{2} \mid 0<g^{m-1} \leq 8 / \sqrt{B} \text { and } g^{\prime} \leq 0 \text { on }\left(z_{2}, z\right)\right\} \leq \infty,
$$

which is again meaningful since $g^{m-1}\left(z_{2}\right)=8 / \sqrt{B}$ and $g^{\prime}\left(z_{2}\right)<0$ by 5.19). For $z \in$ $\left(z_{2}, z_{3}\right)$ we estimate $\Phi(g)$, rather than using Lemma 5.1] according to

$$
\Phi(g)=-\frac{m}{(m-1)^{2}} g+\frac{B}{m-1} g^{m}-A^{2 m-2} g^{2 m-1} \leq \frac{B}{m-1} g^{m-1} g \leq \frac{8 \sqrt{B}}{m-1} g
$$


and thereby deduce from 5.12, recalling that $\frac{y_{0}^{2}}{2} e^{-2 z} \leq 1$ for $z>z_{2}>z_{1}$,

$$
g^{\prime \prime} \leq-\left(\frac{m+1}{m-1}+\frac{y_{0}^{2}}{2} e^{-2 z}\right) g^{\prime}+\frac{8 \sqrt{B}}{m-1} g \leq-\frac{2 m}{m-1} g^{\prime}+\frac{8 \sqrt{B}}{m-1} g \quad \text { on }\left(z_{2}, z_{3}\right) .
$$

Since trivially $\frac{1}{4}\left(\frac{2 m}{m-1}\right)^{2}+\frac{8 \sqrt{B}}{m-1}>0$, Lemma 3.2 states that

$$
\begin{aligned}
g(z) \leq & \frac{g^{\prime}\left(z_{2}\right)-\theta^{-} g\left(z_{2}\right)}{\theta^{+}-\theta^{-}} e^{\theta^{+}\left(z-z_{2}\right)} \\
& +\frac{\theta^{+} g\left(z_{2}\right)-g^{\prime}\left(z_{2}\right)}{\theta^{+}-\theta^{-}} e^{\theta^{-}\left(z-z_{2}\right)}, \quad z \in\left(z_{2}, z_{3}\right),
\end{aligned}
$$

with

$$
\theta^{ \pm}=-\frac{m}{m-1} \pm \sqrt{\left(\frac{m}{m-1}\right)^{2}+\frac{8 \sqrt{B}}{m-1}} .
$$

We claim that if $B$ is sufficiently large and $\varepsilon<\varepsilon_{0}(B)$ then furthermore

$$
g^{\prime}\left(z_{2}\right)<\theta^{-} g\left(z_{2}\right) \text {. }
$$

In view of the eventual dominance of the first term on the right of 5.21, this will on the one hand prove that $z_{3}$ is finite, so that by definition of $z_{3}$, either $g\left(z_{3}\right)=0$ or $g^{\prime}\left(z_{3}\right)=0$. But on the other hand, Lemma 3.2 also asserts that 5.22 entails $g^{\prime}(z) \leq \theta^{-} g(z)$ for all $z \in\left[z_{2}, z_{3}\right]$. Evaluating this at $z=z_{3}$ and noting that $\theta^{-}<0$, we infer that $g\left(z_{3}\right)$ cannot be positive and hence $g$ reaches zero at $z_{3}(B, \varepsilon)$ for such $B$ and $\varepsilon$, which is the desired contradiction to our hypothesis $z_{\infty}=\infty$.

In order to prove (5.22) for

$$
B>B_{6}:=\max \left\{B_{5}, \frac{m^{4}}{64(m-1)^{2}},\left[\frac{4(4+\sqrt{8}) 8^{1 /(m-1)}}{\sqrt{m-1}}\right]^{4(m-1) /(m+1)}\right\},
$$

let us first observe that if $B>\frac{m^{4}}{64(m-1)^{2}}$ then $\left(\frac{m}{m-1}\right)^{2} \leq \frac{8 \sqrt{B}}{m-1}$ and thus

$$
\theta^{-} \geq-\sqrt{\frac{8 \sqrt{B}}{m-1}}-\sqrt{\frac{16 \sqrt{B}}{m-1}}=-\frac{(4+\sqrt{8})}{\sqrt{m-1}} B^{1 / 4} .
$$

Therefore $(5.20)$ yields

$$
\begin{aligned}
g^{\prime}\left(z_{2}\right)-\theta^{-} g\left(z_{2}\right) & =-G^{\prime}\left(z_{2}\right)-\theta^{-}\left(\frac{8}{\sqrt{B}}\right)^{1 /(m-1)} \\
& \leq-\frac{\sqrt{B}}{4}+\frac{(4+\sqrt{8}) 8^{1 /(m-1)}}{\sqrt{m-1}} B^{1 / 4-1 / 2(m-1)} \\
& =\frac{\sqrt{B}}{4}\left[1-\frac{4(4+\sqrt{8}) 8^{1 /(m-1)}}{\sqrt{m-1}} B^{-m+1 / 4(m-1)}\right]<0
\end{aligned}
$$

whenever $B>B_{6}$. 


\subsection{Shooting forwards}

In demonstrating our forward shooting procedure, we again attempt to avoid an accumulation of difficulties and therefore begin by providing an explicit supersolution of a differential equation that will occur below.

Lemma 5.4. Let $M, K_{1}, K_{2}>0, \beta \in(1,2)$ and $\gamma \in(2 \beta-2,2)$. Then there exist positive constants $z_{1}, \delta, c_{1}, c_{2}$ and $\eta_{0}$ such that the function

$$
\psi(z):= \begin{cases}1-e^{-2 z}, & 0 \leq z<z_{1} \\ c_{1} e^{\delta e^{\gamma z}}-c_{2}, & z_{1} \leq z<\infty\end{cases}
$$

belongs to $W_{\mathrm{loc}}^{2, \infty}([0, \infty))$ and satisfies

$$
\mathcal{L} \psi \equiv \psi^{\prime \prime}-\left[M\left(e^{2 z}-1\right)-1-K_{1} \eta e^{\beta z}\right] \psi^{\prime}+\left[M+K_{2} \eta e^{2 \beta z}\right] \psi \leq 0
$$

for all $\eta \in\left(0, \eta_{0}\right]$ and all $z \in(0, \infty), z \neq z_{1}$.

Proof. We pick any $\delta \in(0,2 / \gamma)$ satisfying

$$
\delta \leq\left(\frac{2}{\gamma}\right)^{(2-\gamma) / 4}\left(\frac{M}{8 \gamma}\right)^{(\gamma+2) / 4}
$$

and

$$
\delta \leq \frac{2}{\gamma}\left(\frac{M}{8(M+\gamma+1)}\right)^{(\gamma+2) / 2}
$$

and set

$$
z_{1}:=\frac{1}{\gamma+2} \ln \frac{2}{\delta \gamma} .
$$

Then $z_{1}>0$, and requiring that $\psi$ and $\psi^{\prime}$ be continuous at $z_{1}$ is equivalent to saying

$$
1-e^{-2 z_{1}}=c_{1} e^{\delta e^{\gamma z_{1}}}-c_{2} \text { and } 2 e^{-2 z_{1}}=c_{1} e^{\gamma z_{1}} e^{\delta e^{\gamma z_{1}}},
$$

so that we define

$$
c_{1}:=e^{-\delta e^{\gamma z_{1}}} \quad \text { and } \quad c_{2}:=e^{-2 z_{1}} .
$$

To make the list of our definitions complete, we choose $\eta_{0}>0$ small enough such that

$$
\eta_{0} \leq \frac{2}{2 K_{1} e^{\beta z_{1}}+K_{2} e^{(2 \beta+2) z_{1}}}
$$

and

$$
\eta_{0} \leq \frac{M \delta \gamma}{4\left(K_{1} \delta \gamma+K_{2}\right)} .
$$


With these choices, for $z<z_{1}$ we have $\psi(z)=1-e^{-2 z}, \psi^{\prime}(z)=2 e^{-2 z}$ and $\psi^{\prime \prime}(z)=$ $-4 e^{-2 z}$ and hence, using 5.28 ,

$$
\begin{aligned}
\mathcal{L} \psi= & -4 e^{-2 z}-2\left[M\left(e^{2 z}-1\right)-1-K_{1} \eta e^{\beta z}\right] e^{-2 z} \\
& +\left[M+K_{2} \eta e^{2 \beta z}\right]\left(1-e^{-2 z}\right) \\
= & -M\left(1-e^{-2 z}\right)-2 e^{-2 z}+\eta\left(2 K_{1} e^{(\beta-2) z}+K_{2} e^{2 \beta z}-K_{2} e^{(2 \beta-2) z}\right) \\
= & -M\left(1-e^{-2 z}\right)-\left[2-\eta\left(2 K_{1} e^{\beta z}+K_{2} e^{(2 \beta+2) z}\right)\right] e^{-2 z}-\eta K_{2} e^{(2 \beta-2) z} \\
\leq & -\left[2-\eta_{0}\left(2 K_{1} e^{\beta z_{1}}+K_{2} e^{(2 \beta+2) z_{1}}\right)\right] e^{-2 z} \\
\leq & 0 \quad \text { for all } z<z_{1}
\end{aligned}
$$

whenever $\eta \leq \eta_{0}$.

In the region $z>z_{1}$, however, we compute

$$
\begin{aligned}
\psi(z) & =c_{1} e^{\delta e^{\gamma z}}-c_{2}, \\
\psi^{\prime}(z) & =c_{1} \delta \gamma e^{\gamma z} e^{\delta e^{\gamma z}} \\
\psi^{\prime \prime}(z) & =c_{1}\left(\delta^{2} \gamma^{2} e^{2 \gamma z}+\delta \gamma^{2} e^{\gamma z}\right) e^{\delta e^{\gamma z}}
\end{aligned}
$$

and thereby obtain

$$
\begin{aligned}
\mathcal{L} \psi= & c_{1}\left\{\delta^{2} \gamma^{2} e^{2 \gamma z}+\delta \gamma^{2} e^{\gamma z}-\left[M\left(e^{2 z}-1\right)-1-K_{1} \eta e^{\beta z}\right] \delta \gamma e^{\gamma z}+\left[M+K_{2} \eta e^{2 \beta z}\right]\right\} e^{\delta e^{\gamma z}} \\
& -c_{2}\left[M+K_{2} \eta e^{2 \beta z}\right] \quad \text { for all } z>z_{1} .
\end{aligned}
$$

Dropping the last term, we obtain

$$
\begin{aligned}
\mathcal{L} \psi \leq & c_{1}\left\{\delta^{2} \gamma^{2} e^{-(2-\gamma) z}-M \delta \gamma+(M+\gamma+1) \delta \gamma e^{-2 z}+M e^{-(\gamma+2) z}\right. \\
& \left.+K_{1} \eta \delta \gamma e^{-(2-\beta) z}+K_{2} \eta e^{-(\gamma+2-2 \beta) z}\right\} e^{(\gamma+2) z} e^{\delta e^{\gamma z}} \\
= & : c_{1}\left\{I_{1}-I_{2}+I_{3}+I_{4}+I_{5}+I_{6}\right\} e^{(\gamma+2) z} e^{\delta e^{\gamma z}} \text { for } z>z_{1} .
\end{aligned}
$$

Here,

$$
\begin{aligned}
\frac{I_{4}}{\frac{1}{2} I_{2}} & =\frac{M e^{-(\gamma+2) z}}{\frac{1}{2} M \delta \gamma}=\frac{2}{\delta \gamma} e^{-(\gamma+2) z} \\
& \leq \frac{2}{\delta \gamma} e^{-(\gamma+2) z_{1}}=1 \quad \text { for all } z>z_{1}
\end{aligned}
$$

due to (5.27). Moreover,

$$
\begin{aligned}
\frac{I_{1}}{\frac{1}{8} I_{2}} & =\frac{\delta^{2} \gamma^{2} e^{-(2-\gamma) z}}{\frac{1}{8} M \delta \gamma} \leq \frac{8 \delta \gamma}{M} e^{-(2-\gamma) z_{1}} \\
& =\frac{8 \delta \gamma}{M}\left(\frac{\delta \gamma}{2}\right)^{(2-\gamma) /(\gamma+2)}=\frac{8 \gamma}{M}\left(\frac{\gamma}{2}\right)^{(2-\gamma) /(\gamma+2)} \delta^{4 /(\gamma+2)} \\
& \leq \frac{8 \gamma}{M}\left(\frac{\gamma}{2}\right)^{(2-\gamma) /(\gamma+2)}\left(\frac{2}{\gamma}\right)^{(2-\gamma) /(\gamma+2)} \frac{M}{8 \gamma}=1 \quad \text { for all } z>z_{1}
\end{aligned}
$$


by 5.25 , while according to 5.26 we have

$$
\begin{aligned}
\frac{I_{3}}{\frac{1}{8} I_{2}} & =\frac{(M+\gamma+1) \delta \gamma e^{-2 z}}{\frac{1}{8} M \delta \gamma} \leq \frac{8(M+\gamma+1)}{M} e^{-2 z_{1}} \\
& =\frac{8(M+\gamma+1)}{M}\left(\frac{\delta \gamma}{2}\right)^{2 /(\gamma+2)} \\
& \leq \frac{8(M+\gamma+1)}{M}\left(\frac{\gamma}{2}\right)^{2 /(\gamma+2)}\left(\frac{2}{\gamma}\right)^{2 /(\gamma+2)} \frac{M}{8(M+\gamma+1)} \\
& =1 \quad \text { for all } z>z_{1} .
\end{aligned}
$$

Finally, since $\gamma<2 \beta-2$, from 5.29 we obtain

$$
\begin{aligned}
\frac{I_{5}+I_{6}}{\frac{1}{4} I_{2}} & =\frac{K_{1} \eta \delta \gamma e^{-(2-\beta) z}+K_{2} \eta e^{-(\gamma+2-2 \beta) z}}{\frac{1}{4} M \delta \gamma} \\
& \leq \frac{4\left(K_{1} \delta \gamma+K_{2}\right) \eta}{M \delta \gamma} \leq 1 \quad \text { for all } z>z_{1}
\end{aligned}
$$

whenever $\eta \leq \eta_{0}$. Now 5.24 follows from 5.30-5.34.

We are ready to formulate the outcome of our forward shooting approach in a way appropriate for the proof of Theorem 5.6 below.

Lemma 5.5. Fix $m>1, k>2 / \sqrt{m}, \beta \in(1,2)$ and let

$$
y_{0}:=\sqrt{2} \sqrt{k m A^{m-1}-\frac{2 m}{m-1}}
$$

be as in Lemma 5.3. Then there is $\eta>0$ with the property that for all $z_{1}>0$ there exists $\varepsilon_{1}>0$ such that for any $\varepsilon<\varepsilon_{1}$, the solution of the forward shooting problem

$$
\left\{\begin{array}{l}
g_{f}^{\prime \prime}=\left(\frac{m+1}{m-1}+\frac{y_{0}^{2}}{2} e^{2 z}\right) g_{f}^{\prime}-k m A^{m-1} g_{f+}^{m-1} g_{f}^{\prime}+\Phi\left(g_{f}\right), \quad z>0 \\
g_{f}(0)=1, \quad g_{f}^{\prime}(0)=\varepsilon
\end{array}\right.
$$

satisfies

$$
1 \leq g_{f}^{m-1}(z) \leq 2 \quad \text { for all } z \in\left(0, z_{1}\right)
$$

but

$$
g_{f}^{m-1}\left(z_{2}\right) \geq 1+\eta e^{\beta z_{2}}
$$

with some $z_{2}=z_{2}(\varepsilon)>z_{1}$. 
Proof. We again write $B:=k m A^{m-1}$ and $g$ instead of $g_{f}$. We set

$$
M:=B-\frac{2 m}{m-1}>0, \quad K_{1}:=B, \quad K_{2}:=\frac{|3 m-2| B}{m-1}+\frac{(2 m-1)\left(B-\frac{m}{m-1}\right)}{m-1} .
$$

We fix some $\gamma \in(2 \beta-2,2)$ and then pick any $\eta \in\left(0, \min \left\{\eta_{0}, 1\right\}\right)$, where $\eta_{0}$ is as in Lemma 5.4. Making use of the continuous dependence of $g$ on $\varepsilon$, we can choose some $\varepsilon_{1}>0$ such that whenever $\varepsilon<\varepsilon_{1}$, we have

$$
g^{m-1} \leq 1+\eta / 2 \quad \text { in }\left(0, z_{1}\right)
$$

For $\varepsilon<\varepsilon_{1}$, we now define

$$
\begin{aligned}
& z_{2} \equiv z_{2}(\varepsilon):=\sup \left\{\tilde{z}>0 \mid 1 \leq g^{m-1}(z) \leq 1+\eta e^{\beta z}\right. \text { and } \\
& \left.\qquad g^{\prime}(z) \geq 0 \text { for all } z \in(0, \tilde{z})\right\} \leq \infty
\end{aligned}
$$

noting that this is meaningful because $g(0)=1$ and $g^{\prime}(0)>0$. If $z \in\left(0, z_{2}\right)$ then from the concavity of $\Phi$ on $[1, \infty)$, as asserted by Lemma 5.1 , we have the estimate

$$
\begin{aligned}
\Phi(g) & \geq \Phi^{\prime}\left(\left(1+\eta e^{\beta z}\right)^{1 /(m-1)}\right)(g-1) \\
& =\left\{-\frac{m}{(m-1)^{2}}+\frac{k m^{2} A^{m-1}}{m-1}\left(1+\eta e^{\beta z}\right)-(2 m-1) A^{2 m-2}\left(1+\eta e^{\beta z}\right)^{2}\right\}(g-1) .
\end{aligned}
$$

Since $A^{2 m-2}=\frac{B}{m-1}-\frac{m}{(m-1)^{2}}$ due to 2.5 , for such $z$ we obtain

$$
\begin{aligned}
\Phi(g) & \geq\left\{\frac{2 m}{m-1}-B-\left[\frac{(3 m-2) B}{m-1}-\frac{2 m(2 m-1)}{(m-1)^{2}}\right] \eta e^{\beta z}\right. \\
& \geq\left\{\frac{2 m}{m-1}-B-K_{2} \eta e^{2 \beta z}\right\}(g-1)
\end{aligned}
$$

because $\eta<1$ and $e^{\beta z} \geq 1$. Therefore the function $G:=g-1$ satisfies

$$
\begin{aligned}
G^{\prime \prime}= & \left(\frac{m+1}{m-1}+\frac{y_{0}^{2}}{2} e^{2 z}\right) G^{\prime}-B g^{m-1} G^{\prime}+\Phi(g) \\
\geq & \left(\frac{m+1}{m-1}+\frac{y_{0}^{2}}{2} e^{2 z}\right) G^{\prime}-B\left(1+\eta e^{\beta z}\right) G^{\prime}-\left(B-\frac{2 m}{m-1}+K_{2} \eta e^{2 \beta z}\right) G \\
= & \left(\frac{m+1}{m-1}+\left(B-\frac{2 m}{m-1}\right) e^{2 z}-B-B \eta e^{\beta z}\right) G^{\prime} \\
& -\left(B-\frac{2 m}{m-1}+K_{2} \eta e^{2 \beta z}\right) G \\
= & {\left[M\left(e^{2 z}-1\right)-1-K_{1} \eta e^{\beta z}\right] G^{\prime}-\left[M+K_{2} \eta e^{2 \beta z}\right] G, \quad z \in\left(0, z_{2}\right), }
\end{aligned}
$$


according to our definitions of $y_{0}, M, K_{1}$ and $K_{2}$. Applying Lemma 3.1 to $\varphi:=G / \varepsilon$ and the positive function $\psi$ provided by Lemma 5.4 we conclude that $G \geq \varepsilon \psi$ and $G^{\prime} / G \geq \psi^{\prime} / \psi$ on $\left(0, z_{2}\right)$. Since $e^{-\beta z /(m-1)} \psi(z) \rightarrow \infty$ as $z \rightarrow \infty$, we infer that for any $\varepsilon<\varepsilon_{1}$ the number $z_{2}=z_{2}(\varepsilon)$ is finite and $G^{\prime}\left(z_{2}\right)>0$, so that $g^{m-1}\left(z_{2}\right)=1+\eta e^{\beta z_{2}}$. In particular, $z_{2}>z_{1}$ in view of (5.38) and thereby (5.36) and (5.37) are proved.

\subsection{Existence of solutions with local minima}

The following theorem combines the results of the preceding two subsections and thereby proves that if the shooting parameter $\varepsilon>0$ above is chosen small and equal in both shooting directions then we can glue together the resulting forward and backward functions and thereby, adding one further continuous dependence argument, obtain a solution of 2.1], (2.2) with a positive local minimum.

Theorem 5.6. Let $m>1$. Then there exists $k_{0}>2 / \sqrt{m}$ such that for all $k \geq k_{0}$ there is $\sigma_{1}>0$ such that the solution $w_{\sigma_{1}}$ of 2.1, 2.2) attains a positive local minimum somewhere in $(0, \infty)$.

Proof. Planning to apply Lemmas 5.3 and 5.5, we set again

$$
y_{0}:=\sqrt{2} \sqrt{k m A^{m-1}-\frac{2 m}{m-1}} \equiv \sqrt{\frac{k^{2} m^{2}-4 m+\sqrt{k^{2} m^{2}-4 m}}{m-1}} .
$$

We take $k_{0}$ as generated by Lemma 5.3 and then, given any $k \geq k_{0}$, let $\varepsilon_{0}$ be as provided by the same lemma. Fixing an arbitrary $\beta \in(1,2)$, we take $\eta>0$ from Lemma 5.5, then choose $z_{1}>0$ large enough such that

$$
e^{z_{1}}>\frac{2 A^{m-1}}{y_{0} \mu^{m-1}}
$$

and

$$
e^{(\beta-1) z_{1}}>\frac{y_{0} \mu^{m-1}}{\eta A^{m-1}},
$$

and let $\varepsilon_{1}$ and $z_{2}>z_{1}$ denote the numbers furnished by Lemma 5.5. We now pick any $\varepsilon \in\left(0, \min \left\{\varepsilon_{0}, \varepsilon_{1}\right\}\right)$ and let $g_{b}$ and $g_{f}$ be the corresponding solutions of 5.3$)$ and 5.47 , respectively, with $g_{b}(0)=g_{f}(0)=1, g_{b}^{\prime}(0)=-\varepsilon$ and $g_{f}^{\prime}(0)=\varepsilon$. Then

$$
\tilde{f}(y):= \begin{cases}g_{b}\left(-\ln \frac{y}{y_{0}}\right), & y \in\left(0, y_{0}\right), \\ g_{f}\left(\ln \frac{y}{y_{0}}\right), & y \in\left[y_{0}, \infty\right),\end{cases}
$$

defines a continuously differentiable function $\tilde{f}$ that, according to the considerations at the beginning of the present section, solves 5.1. Hence, $\tilde{f}$ is actually smooth and $\tilde{w}:=$ $\tilde{f} w_{s}^{+}$is a solution of 2.1 . 
According to Lemma 5.3

$$
\tilde{w} \text { has a zero in }\left(0, y_{0}\right) \text {. }
$$

Due to Lemma 5.5. we furthermore have $g_{f}^{m-1}(z) \leq 2$ for all $z \leq z_{1}$, and in particular

$$
\frac{\tilde{w}^{m-1}\left(y_{0} e^{z_{1}}\right)}{\mu^{m-1}}=\frac{g_{f}^{m-1}\left(z_{1}\right) A^{m-1}}{e^{z_{1}} y_{0} \mu^{m-1}} \leq \frac{2 A^{m-1}}{e^{z_{1}} y_{0} \mu^{m-1}}<1
$$

because of 5.39 . On the other hand, Lemma 5.5 also says that $g_{f}^{m-1}\left(z_{2}\right) \geq 1+\eta e^{\beta z_{2}}>$ $\eta e^{\beta z_{2}}$, so that

$$
\frac{\tilde{w}^{m-1}\left(y_{0} e^{z_{2}}\right)}{\mu^{m-1}}=\frac{g_{f}^{m-1}\left(z_{2}\right) A^{m-1}}{e^{z_{2}} y_{0} \mu^{m-1}}>\frac{\eta A^{m-1} e^{(\beta-1) z_{2}}}{y_{0} \mu^{m-1}}>\frac{\eta A^{m-1} e^{(\beta-1) z_{1}}}{y_{0} \mu^{m-1}}>1
$$

in view of 5.40). Finally, from the definitions of $\mu$ and $y_{0}$ and the fact that $g_{b}(0)=$ $g_{f}(0)=1$ we have

$$
\begin{aligned}
A^{2(m-1)}\left(\tilde{w}^{-2(m-1)}\left(y_{0}\right)-\mu^{-2(m-1)}\right)= & A^{2(m-1)}\left(\left(w_{s}^{+}\left(y_{0}\right)\right)^{-2(m-1)}-\mu^{-2(m-1)}\right) \\
= & y_{0}^{2}-\left(\frac{A}{\mu}\right)^{2(m-1)} \\
= & \frac{k^{2} m^{2}-4 m+\sqrt{k^{2} m^{2}-4 m}}{m-1} \\
& -\frac{k^{2} m^{2}+k m \sqrt{k^{2} m^{2}-4 m}-2 m}{m-1} \\
< & -\frac{2 m}{m-1} \quad \text { if } k>\frac{1}{m},
\end{aligned}
$$

whence altogether we conclude, writing $y_{1}:=y_{0} e^{z_{1}}$ and $y_{2}:=y_{0} e^{z_{2}}$, that $y_{0}<y_{1}<y_{2}$ and

$$
\tilde{w}\left(y_{0}\right)>\mu, \quad \tilde{w}\left(y_{1}\right)<\mu, \quad \tilde{w}\left(y_{2}\right)>\mu .
$$

Therefore $\tilde{w}$ attains a-necessarily positive-local minimum at some $\tilde{y} \in\left(y_{0}, y_{2}\right)$. In order to derive from this result the existence of a solution of 2.1), 2.2. having a minimum at $\tilde{y}$, we introduce the value attained at $\tilde{y}$ as a parameter and apply one further continuous dependence argument. More precisely, for $a \in(0, \mu]$ we let $\tilde{w}_{a}$ denote the solution of 2.1 with $\tilde{w}_{a}(\tilde{y})=a$ and $\tilde{w}_{a}^{\prime}(\tilde{y})=0$, so that the function $\tilde{w}$ constructed above coincides with $\tilde{w}_{a}$ when $a=a_{0}:=\tilde{w}(\tilde{y})$. Therefore, due to 5.41, the set

$$
\mathcal{A}:=\left\{a \in(0, \mu] \mid \tilde{w}_{a} \text { has a zero in }(0, \tilde{y})\right\}
$$

is not empty and hence $a_{1}:=\sup \mathcal{A}$ is well-defined. Since $w \equiv \mu$ solves $(2.1)$, the number $\mu$ does not belong to $\mathcal{A}$ and thus, by continuous dependence of $\tilde{w}_{a}$ on $a$ in $C^{0}([0, \tilde{y}])$, we have $a_{1}<\mu$. We claim that

$$
\tilde{w}_{a_{1}}>0 \quad \text { in }(0, \tilde{y}) \quad \text { and } \quad \tilde{w}_{a_{1}}(0)=0 .
$$


In fact, if $\tilde{w}_{a_{1}}$ had a zero in $(0, \tilde{y})$ then, by $\left.\sqrt{2.1}\right), \tilde{w}_{a_{1}}$ should change sign in $(0, \tilde{y})$, so that, again due to continuous dependence on $a$, the same is true for some $\tilde{w}_{a}$ with $a \in\left(a_{1}, \mu\right)$, contradicting the definition of $a_{1}$. On the other hand, it is impossible that $\tilde{w}_{a_{1}}$ is positive throughout $[0, \tilde{y}]$, because then also $\tilde{w}_{a}$ would be positive whenever $a$ is sufficiently close to $a_{1}$, whence $\sup \mathcal{A}$ would be smaller than $a_{1}$.

Having shown 5.42, we set $\sigma_{1}:=\tilde{w}_{a_{1}}^{\prime}(0)$. Then, due to the uniqueness properties of 2.1, $\sigma_{1}$ is positive and $w_{\sigma_{1}} \equiv \tilde{w}_{a_{1}}$. Thus, $w_{\sigma_{1}}$ satisfies $w_{\sigma_{1}}^{\prime}(\tilde{y})=0$ and $w_{\sigma_{1}}(\tilde{y}) \in(0, \mu)$ and therefore, by $\sqrt{2.1}, w_{\sigma_{1}}^{\prime \prime}(\tilde{y})>0$, so that indeed $w_{\sigma_{1}}$ attains a local minimum at $\tilde{y}$.

\section{Proof of Theorem 1.1}

Reviewing Theorems 4.3 and 5.6 one can expect that there is an intermediate value of $\sigma$ for which the function $w_{\sigma}$ solving (2.1), 2.2) remains positive in $(0, \infty)$. In fact, starting from the solution $w_{\sigma_{1}}$ with a local minimum, one can continuously deform this solution by diminishing $\sigma$, and ask what might happen to the local minimum point of $w_{\sigma}$, say $y(\sigma)$, when $\sigma$ decreases from $\sigma_{1}$ to $\sigma_{0}$, where $\sigma_{0}$ is as in Theorem 4.3 Loosely speaking, this minimum must disappear at some $\sigma$ between $\sigma_{0}$ and $\sigma_{1}$, and the only conceivable possibility for this to occur is that $y(\sigma) \rightarrow \infty$ when $\sigma$ approaches this critical value. These considerations form the core of the proof of our main result.

Proof of Theorem 1.1. We first make sure that if $k$ is large then, for some appropriate $\bar{\sigma}>0$, the solution $w_{\bar{\sigma}}$ of 2.1], 2.2) remains positive throughout $(0, \infty)$.

For this purpose, we observe that according to Theorem 5.6, the set

$$
\Sigma:=\left\{\sigma>0 \mid w_{\sigma} \text { has a local minimum in }(0, \infty)\right\}
$$

is not empty if $k \geq k_{0}$ for some sufficiently large $k_{0}>2 / \sqrt{m}$. For $\sigma \in \Sigma$, let

$$
y(\sigma):=\inf \left\{y \in(0, \infty) \mid w_{\sigma} \text { has a local minimum at } y\right\}
$$

and define the number

$$
\bar{\sigma}:=\inf \Sigma,
$$

which is positive due to the result of Theorem 4.3 . To prove that $\bar{\sigma}$ has the desired property, we fix a sequence of numbers $\sigma_{j} \in \Sigma$ such that $\sigma_{j} \rightarrow \bar{\sigma}$ as $j \rightarrow \infty$, and claim that

$$
y\left(\sigma_{j}\right) \rightarrow \infty \quad \text { as } j \rightarrow \infty ;
$$

once this has been shown, the assertion immediately follows from the continuous dependence on $\sigma$ of the solutions of 2.1, 2.2): Namely, (6.1) implies that $w_{\bar{\sigma}}(y)=$ $\lim _{j \rightarrow \infty} w_{\sigma_{j}}(y)$ is nonnegative for all $y>0$ and hence even positive for each $y>0$, because $w_{\bar{\sigma}} \not \equiv 0$.

To show (6.1), let us proceed by contradiction. Suppose that $y\left(\sigma_{j}\right) \rightarrow \bar{y}_{0} \in[0, \infty)$ for a subsequence. Then, since $w_{\sigma_{j}} \rightarrow w_{\bar{\sigma}}$ in $C^{1}\left(\left[0, \bar{y}_{0}+1\right]\right)$, we infer that $w_{\bar{\sigma}}^{\prime}\left(\bar{y}_{0}\right)=$ $\lim _{j \rightarrow \infty} w_{\sigma_{j}}^{\prime}\left(y\left(\sigma_{j}\right)\right)=0$ and $w_{\bar{\sigma}}\left(\bar{y}_{0}\right)=\lim _{j \rightarrow \infty} w_{\sigma_{j}}\left(y\left(\sigma_{j}\right)\right) \in[0, \mu]$. But $w_{\bar{\sigma}} \not \equiv 0$ and 
$w_{\bar{\sigma}} \not \equiv \mu$, whence actually $w_{\bar{\sigma}}\left(\bar{y}_{0}\right) \in(0, \mu)$. Thus, again by continuous dependence, we can find positive numbers $\nu_{1}$ and $\nu_{2}$ such that whenever $|\sigma-\bar{\sigma}|<v_{1}$ and $\left|y-\bar{y}_{0}\right|<v_{2}$, we have $w_{\sigma}(y) \in(0, \mu)$ and $w_{\sigma}^{\prime}\left(\bar{y}_{0}-v_{2}\right)>0>w_{\sigma}^{\prime}\left(\bar{y}_{0}+v_{2}\right)$. This implies that each such $w_{\sigma}$ attains a local minimum in $\left(\bar{y}_{0}-v_{2}, \bar{y}_{0}+v_{2}\right)$, evidently contradicting the definition of $\bar{\sigma}$.

Next we show that $w:=w_{\bar{\sigma}}$ satisfies

$$
w(y) \searrow 0 \quad \text { as } y \rightarrow \infty .
$$

Indeed, from Lemma 4.2 we already know that $w^{\prime}(y)<0$ for all sufficiently large $y$, so that $w(y) \searrow w_{\infty}$ as $y \rightarrow \infty$, with some $w_{\infty} \geq 0$. In view of Lemma 2.2, either $w_{\infty}=0$ or $w_{\infty}=\mu$, whence it remains to exclude the possibility that $w(y) \searrow \mu$ as $y \rightarrow \infty$. If this were true then $v:=w-\mu$ would be eventually positive and decrease to zero as $y \rightarrow \infty$. Using 2.1) and the convexity of $s \mapsto s^{2 m-1}$ on $(0, \infty)$, we see that

$$
\begin{aligned}
v^{\prime \prime} & =\frac{y}{2} v^{\prime}-k m w^{m-1} v^{\prime}+\frac{1}{2(m-1)}(\mu+v)-(\mu+v)^{2 m-1} \\
& \leq \frac{y}{2} v^{\prime}-k m w^{m-1} v^{\prime}+\frac{1}{2(m-1)} \mu+\frac{1}{2(m-1)} v-\mu^{2 m-1}-(2 m-1) \mu^{2 m-2} v \\
& =\left(\frac{y}{2}-k m w^{m-1}\right) v^{\prime}-v<0
\end{aligned}
$$

for all sufficiently large $y$, because $w$ is bounded and, eventually, $v^{\prime}<0$ and $v>0$. Thus, $v$ should be concave for large $y$, which is incompatible with the assumption that $v(y) \searrow 0$. Consequently, $w_{\infty}=0$ and 6.2 has been established.

Now we claim that for all $\alpha<1 /(m-1)$ there exist $y_{\alpha}>0$ and $C_{\alpha}>0$ such that

$$
w(y) \leq C_{\alpha} y^{-\alpha} \quad \text { for all } y \geq y_{\alpha} .
$$

To prove this, we let $\alpha$ be given and choose $y_{\alpha}>0$ large enough such that

$$
\begin{aligned}
& y_{\alpha}^{2} \geq \frac{4}{\frac{1}{m-1}-\alpha}(\alpha-1)(\alpha-2), \\
& y_{\alpha}^{2} \geq 4(1-\alpha), \\
& w^{\prime}(y) \leq 0 \text { for all } y \geq y_{\alpha}, \\
& w(y) \leq\left(\frac{\frac{1}{m-1}-\alpha}{4}\right)^{1 / 2(m-1)} \quad \text { for all } y \geq y_{\alpha},
\end{aligned}
$$

where we note that 6.6 and 6.7 are possible due to 6.2. We now multiply 2.1 by $y^{\alpha-1}$ and integrate over $\left(y_{\alpha}, y\right), y>y_{\alpha}$, to obtain

$$
\begin{aligned}
& \frac{1}{2} \int_{y_{\alpha}}^{y} \xi^{\alpha} w^{\prime}(\xi) d \xi+\frac{1}{2(m-1)} \int_{y_{\alpha}}^{y} \xi^{\alpha-1} w(\xi) d \xi \\
& =\int_{y_{\alpha}}^{y} \xi^{\alpha-1} w^{\prime \prime}(\xi) d \xi+k \int_{y_{\alpha}}^{y} \xi^{\alpha-1}\left(w^{m}\right)^{\prime}(\xi) d \xi+\int_{y_{\alpha}}^{y} \xi^{\alpha-1} w^{2 m-1}(\xi) d \xi
\end{aligned}
$$


After integration by parts, the left-hand side becomes

$$
\begin{aligned}
\frac{1}{2} \int_{y_{\alpha}}^{y} \xi^{\alpha} w^{\prime} & (\xi) d \xi+\frac{1}{2(m-1)} \int_{y_{\alpha}}^{y} \xi^{\alpha-1} w(\xi) d \xi \\
= & \frac{1}{2} y^{\alpha} w(y)-\frac{1}{2} y_{\alpha}^{\alpha} w\left(y_{\alpha}\right)+\frac{1}{2}\left(\frac{1}{m-1}-\alpha\right) \int_{y_{\alpha}}^{y} \xi^{\alpha-1} w(\xi) d \xi
\end{aligned}
$$

On the right-hand side of (6.8), the second term is nonpositive due to $(6.6)$, whereas in the first term we integrate by parts twice and use $6.4-(6.6)$ to see that

$$
\begin{aligned}
\int_{y_{\alpha}}^{y} \xi^{\alpha-1} w^{\prime \prime}(\xi) d \xi= & -(\alpha-1) \int_{y_{\alpha}}^{y} \xi^{\alpha-2} w^{\prime}(\xi) d \xi+y^{\alpha-1} w^{\prime}(y)-y_{\alpha}^{\alpha-1} w^{\prime}\left(y_{\alpha}\right) \\
= & (\alpha-1)(\alpha-2) \int_{y_{\alpha}}^{y} \xi^{\alpha-3} w(\xi) d \xi \\
& -(\alpha-1) y^{\alpha-2} w(y)+(\alpha-1) y_{\alpha}^{\alpha-2} w\left(y_{\alpha}\right) \\
& +y^{\alpha-1} w^{\prime}(y)-y_{\alpha}^{\alpha-1} w^{\prime}\left(y_{\alpha}\right) \\
\leq & \frac{1}{4}\left(\frac{1}{m-1}-\alpha\right) \int_{y_{\alpha}}^{y} \xi^{\alpha-1} w(\xi) d \xi \\
& +\frac{1}{4} y^{\alpha} w(y)+(\alpha-1) y_{\alpha}^{\alpha-2} w\left(y_{\alpha}\right)-y_{\alpha}^{\alpha-1} w^{\prime}\left(y_{\alpha}\right) .
\end{aligned}
$$

By (6.7), the last term in 6.8 can be estimated in the following way:

$$
\int_{y_{\alpha}}^{y} \xi^{\alpha-1} w^{2 m-1}(\xi) d \xi \leq \frac{1}{4}\left(\frac{1}{m-1}-\alpha\right) \int_{y_{\alpha}}^{y} \xi^{\alpha-1} w(\xi) d \xi .
$$

In view of 6.9-6.11, 6.8 becomes

$$
\frac{1}{4} y^{\alpha} w(y) \leq \frac{1}{2} y_{\alpha}^{\alpha} w\left(y_{\alpha}\right)+(\alpha-1) y_{\alpha}^{\alpha-2} w\left(y_{\alpha}\right)-y_{\alpha}^{\alpha-1} w^{\prime}\left(y_{\alpha}\right)
$$

and thus 6.3 follows.

Let us now show that

$$
w(y) \leq C y^{1 /(m-1)} \quad \text { for all } y>0
$$

with some $C>0$. To see this, we fix $\alpha<1 /(m-1)$ close to $1 /(m-1)$ such that

$$
\alpha>\frac{1}{m-1}-2 \quad \text { and } \quad \alpha>\frac{1}{(m-1)(2 m-1)}
$$

where the latter is possible since $m>1$ implies $1 /(2 m-1)<1$. Moreover, we let $\hat{y} \geq y_{\alpha}$ be such that

$$
\hat{y}^{2} \geq 4\left(1-\frac{1}{m-1}\right) \text {. }
$$


We now multiply 2.1 by $y^{1 /(m-1)-1}$ and integrate over $(\hat{y}, y)$ with $y>\hat{y}$ to achieve

$$
\begin{aligned}
\frac{1}{2} y^{1 /(m-1)} w(y)- & \frac{1}{2} \hat{y}^{1 /(m-1)} w(\hat{y}) \\
= & \frac{1}{2} \int_{\hat{y}}^{y} \xi^{1 /(m-1)} w^{\prime}(\xi) d \xi+\frac{1}{2(m-1)} \int_{\hat{y}}^{y} \xi^{1 /(m-1)-1} w(\xi) d \xi \\
= & \int_{\hat{y}}^{y} \xi^{1 /(m-1)-1} w^{\prime \prime}(\xi) d \xi+k \int_{\hat{y}}^{y} \xi^{1 /(m-1)-1}\left(w^{m}\right)^{\prime}(\xi) d \xi \\
& +\int_{\hat{y}}^{y} \xi^{1 /(m-1)-1} w^{2 m-1}(\xi) d \xi .
\end{aligned}
$$

Again integrating by parts and dropping nonpositive terms as above, we arrive at

$$
\begin{aligned}
& \frac{1}{2} y^{1 /(m-1)} w(y)-\frac{1}{2} \hat{y}^{1 /(m-1)} w(\hat{y}) \\
& \leq\left(\frac{1}{m-1}-1\right)\left(\frac{1}{m-1}-2\right) \int_{\hat{y}}^{y} \xi^{1 /(m-1)-3} w(\xi) d \xi \\
&-\left(\frac{1}{m-1}-1\right) y^{1 /(m-1)-2} w(y)+\left(\frac{1}{m-1}-1\right) \hat{y}^{1 /(m-1)-2} w(\hat{y}) \\
&-\hat{y}^{1 /(m-1)-1} w^{\prime}(\hat{y})+\int_{\hat{y}}^{y} \xi^{1 /(m-1)-1} w^{2 m-1}(\xi) d \xi .
\end{aligned}
$$

Here, from 6.3 and 6.13) we obtain

$$
\int_{\hat{y}}^{y} \xi^{1 /(m-1)-3} w(\xi) d \xi \leq C_{\alpha} \int_{\hat{y}}^{\infty} \xi^{1 /(m-1)-3-\alpha} d \xi<\infty
$$

and

$$
\int_{\hat{y}}^{y} \xi^{1 /(m-1)-1} w^{2 m-1}(\xi) d \xi \leq C_{\alpha}^{2 m-1} \int_{\hat{y}}^{\infty} \xi^{1 /(m-1)-1-(2 m-1) \alpha} d \xi<\infty,
$$

while 6.14 guarantees that

$$
-\left(\frac{1}{m-1}-1\right) y^{1 /(m-1)-2} w(y) \leq \frac{1}{4} y^{1 /(m-1)} w(y) \quad \text { for all } y \geq \hat{y} .
$$

By these observations, 6.15) turns into

$$
\frac{1}{2} y^{1 /(m-1)} w(y) \leq \bar{C}+\frac{1}{4} y^{1 /(m-1)} w(y) \quad \text { for all } y \geq \hat{y}
$$

with some $\bar{C}>0$. This immediately results in 6.12 , because $w$ is bounded in $(0, \hat{y})$.

Having thus found a positive solution $w$ of (2.1) satisfying [6.12, we define $u$ by (1.2). By 6.12,

$$
\begin{aligned}
u(x, t) & =(T-t)^{-1 / 2(m-1)} w\left((T-t)^{-1 / 2} x\right) \\
& \leq C x^{-1 /(m-1)} \quad \text { for all } x>0 \text { and any } t<T .
\end{aligned}
$$


On the other hand,

$$
\begin{aligned}
u\left((T-t)^{1 / 2}, t\right) & =(T-t)^{-1 / 2(m-1)} w(1) \\
& \rightarrow \infty \quad \text { as } t \searrow T,
\end{aligned}
$$

whereby the proof is complete.

Acknowledgments. The authors were partially supported by the European Community's Human Potential Programme under contract HPRN-CT-2002-00274, Fronts-Singularities. M. Fila also acknowledges the support of the VEGA Grant 1/3021/06. We thank Joost Hulshof and Fred Weissler for many fruitful stimulating discussions.

\section{References}

[1] Aguirre, J., Escobedo, M.: On the blow-up of solutions for a convective reaction diffusion equation. Proc. Roy. Soc. Edinburgh Sect. A 123, 433-460 (1993) Zbl 0801.35038 MR 1226611

[2] Berestycki, H., Lions, P.-L., Peletier, L. A.: An ODE approach to the existence of positive solutions for semilinear problems in $\mathbb{R}^{N}$. Indiana Univ. Math. J. 30, 141-157 (1981) Zbl 0522.35036 MR 0600039

[3] Chen, T.-F., Levine, H. A., Sacks, P. E.: Analysis of a convective reaction-diffusion equation. Nonlinear Anal. 12, 1349-1370 (1988) Zbl 0685.35016 MR 0972404

[4] Fila, M., Filo, J.: Blow-up on the boundary: a survey. In: Singularities and Differential Equations (Warszawa, 1993), Banach Center Publ. 33, Inst. Math., Polish Acad. Sci., Warszawa, 67-78 (1996) Zbl 0858.35065 MR 1449147

[5] Fila, M., Lieberman, G. M.: Derivative blow-up and beyond for quasilinear parabolic equations. Differential Integral Equations 7, 811-821 (1994) Zbl 0811.35059 MR 1270105

[6] Fila, M., Sacks, P. E.: The transition from decay to blow-up in some reaction-diffusionconvection equations. In: World Congress of Nonlinear Analysts '92 (Tampa, FL, 1992), de Gruyter, Berlin, 455-463 (1996) Zbl 0849.35057 MR 1389096

[7] Friedman, A.: Blow up of solutions of nonlinear parabolic equations. In: Nonlinear Diffusion Equations and Their Equilibrium States, I (Berkeley, CA, 1986), Springer, New York, 301318 (1988) Zbl 0669.35047 MR 0956073

[8] Friedman, A., McLeod, J. B.: Blow up of positive solutions of semilinear heat equations. Indiana Univ. Math. J. 34, 425-447 (1985) Zbl 0576.35068 MR 0783924

[9] Giga, Y., Kohn, R. V.: Asymptotically self-similar blowup of semilinear heat equations. Comm. Pure Appl. Math. 38, 297-319 (1985) Zbl 0585.35051 MR 0784476

[10] Henry, D.: Geometric Theory of Semilinear Parabolic Equations. Lecture Notes in Math. 840, Springer, Berlin (1981) Zbl 0456.35001 MR 0610244

[11] Lacey, A. A.: Global blow-up of a nonlinear heat equation. Proc. Roy. Soc. Edinburgh Sect. A 104, 161-167 (1986) Zbl 0627.35047 MR 0877898

[12] Levine, H. A., Payne, L. E., Sacks, P. E., Straughan, B.: Analysis of a convective reaction-diffusion equation II. SIAM J. Math. Anal. 20, 133-147 (1989) Zbl 07052.35126 MR 0977493

[13] Mueller, C. E., Weissler, F. B.: Single point blow-up for a general semilinear heat equation. Indiana Univ. Math. J. 34, 881-913 (1985) Zbl 0597.35057 MR 0808833

[14] Samarskii, A. A., Galaktionov, V. A., Kurdyumov, S. P., Mikhailov, A. P.: Blow-up in Quasilinear Parabolic Equations. de Gruyter, Berlin (1995) Zbl 1020.35001 MR 1330922 
[15] Souplet, Ph.: Gradient blow-up for multidimensional nonlinear parabolic equations with general boundary conditions. Differential Integral Equations 15, 237-256 (2002) Zbl 1015.35016 MR 1870471

[16] Souplet, Ph., Tayachi, S., Weissler, F. B.: Exact self-similar blow-up of solutions of a semilinear parabolic equation with a nonlinear gradient term. Indiana Univ. Math. J. 45, 655-682 (1996) Zbl 0990.35061 MR 1422101

[17] Souplet, Ph., Weissler, F. B.: Self-similar subsolutions and blowup for nonlinear parabolic equations. J. Math. Anal. Appl. 212, 60-74 (1997) Zbl 0892.35011 MR 1460184

[18] Weissler, F. B.: Single-point blowup for a semilinear initial value problem. J. Differential Equations 55, 204-224 (1984) Zbl 0555.35061 MR 0764124 